\title{
Re-investigation of Dark Etching Regions and White Etching Bands in SAE 52100 bearing steel due to Rolling Contact Fatigue
}

\author{
Mostafa El Laithy ${ }^{\mathrm{a}}$, Ling Wang ${ }^{\mathrm{a}}$, Terry J. Harvey ${ }^{\mathrm{a}}$, Bernd Vierneusel ${ }^{\mathrm{b}}$ \\ ${ }^{a}$ Mechanical Engineering Department, University of Southampton, University Road, Southampton SO17 1BJ, \\ $U K$

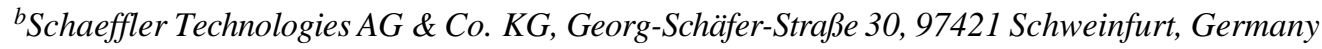

\begin{abstract}
Microstructural alterations such as dark etching regions (DERs) and white etching bands (WEBs) have been known to manifest in the subsurface of steel bearings due to rolling contact fatigue (RCF) under medium-high stress cycles. Even though such manifestations have been reported substantially in literature for decades, their formation and their evolution mechanisms are not fully understood. As part of the re-investigation of DERs and WEBs, this paper presents the results from characterization of DER and WEBs, including both low angle bands (LABs) and high angle bands (HABs), formed in SAE 52100 bearings at different stages of bearing life. Angular contact ball bearings (ACBBs), subjected to RCF testing under different contact pressure, stress cycles and steel cleanliness, have been examined to reveal new information related to the microstructural alteration processes, e.g. the 3-D structure of the WEBs and their growth pattern. Contrary to some of the literature results, the experimental results have shown that the mean depths and density of WEBs (LABs and HABs), as opposed to DER, are correlated with the position and distribution of the principle shear stress. The results also show that HABs form as a consequence of LABs especially in densed LAB areas in the ACBBs studied here.
\end{abstract}

Keywords: Rolling Contact Fatigue; Dark Etching Regions; White Etching Bands; Shear Stress; Steel Bearings

\section{Introduction}

Cyclic stresses experienced by rolling element bearings due to rolling contact fatigue (RCF) has shown to result 
in the manifestation of irreversible microstructural alterations in the subsurface known as dark etching regions (DERs) and white etching bands (WEBs). The names of DER and WEBs were given due to the brightness of the features in steel bearings when etched with Nital giving a 'dark' and 'white' contrast under light optical microscope (LOM) respectively.

DER was first observed in the subsurface of a bearing inner race in 1947 [1] but has been reported under different names since then such as dark etching constituent (DEC) [2], mechanical troostite [1], dark needle [3], low temperature bainite [4] and tempered martensite [5]. DER has been shown to be a consequence of martensite decay in the subsurface of steel bearings which is an implication of microstructural instability $[6,7,8]$. DER has been reported to form in the region of maximum shear stress located in subsurface after a high number of stress cycles (> 5 million cycles) $[3,4,5,8,9,10,11,12]$. DER has been found to develop beyond a certain threshold level of 2.5 GPa contact pressure, which is similar to the shakedown limit of SAE 52100 bearing steel under point contact [13]. It has thus been suggested that stress and plastic deformation play a significant role in the formation of DER $[7,8,14,15]$. DERs appear to be a sickle shape in the subsurface of the ball bearing inner ring when observed in the axial cross section (perpendicular to the rolling direction) corresponding to the contact area between the raceway and rolling element; and as a band that is parallel to the raceway in the circumferential cross section (parallel to the rolling direction).

DER consists of dark patches randomly distributed in the parent martensite matrix with the former consuming the latter with increasing stress cycles. These dark patches have been found to consist of ferrite grains $[2,4,5,7,16$, $17,18,19]$. DER is believed to be formed in a manner similar to over- tempering martensite $[15,20]$ which is supported by the fact that DER has not been observed in fatigue tested bearings initially tempered to a hardness of around 700-720 $\mathrm{HV}[21,22,23]$. It is suggested that DER is caused by the carbon migration from martensite to heavily dislocated regions and nano-sized tempered carbides $[7,8,10,12,17]$. Severe tempering has been proposed to lead to more mature precipitates with poorer coherency between the precipitates and the matrix [24]. It is therefore postulated that increasing the initial tempering temperature and time will result in the loss of coherence of $\varepsilon$-carbides in the steel matrix and thus prevent the formation of DER. The coherency loss is believed to be decisive for the reduced dark etching response of the material due to the reduction of micro strain in the crystallites while more pronounce dark etching is observed in the presence of large micro-strains associated with 
coherent precipitates $[12,15,16,21]$. Reports have also suggested that DER is not found in steel samples with retained austenite content $<2 \%$ [9]. While tests have shown lower retained austenite content in the DER compared to the surrounding matrix, the contribution of retained austenite to DER formation is yet to be established [12, 17].

WEBs are found to form at later stages of the bearing life compared to DER. They have typically been observed to form in dark etching regions but have also been reported to form without DER formation [22, 25], i.e. experimental evidence has shown that WEBs can nucleate either after DER formed in the dark etching regions or directly from the steel matrix without DER preformed. The former has been reported more than the latter in the literature although there has not been much research focused on investigating this. It has been recently proposed that WEBs form in steels with hardness below 720 HV without DER or after DER formation when steel hardness is over $720 \mathrm{HV}$ where material softening occurs however no verified explanation is given [7, 22].

It has been widely reported that, in the axial cross-section of a raceway, WEBs appear to be parallel to the contact surface while in the circumferential cross-section they appear to be inclined to the surface at an angle of 20$35^{\circ}$ and at $65-85^{\circ}$ towards the rolling direction for low angle bands (LABs) and high angle bands (HABs) respectively. LABs are typically found to form beyond 100 million stress cycles followed by HABs which start forming beyond 500 million cycles [5]. LABs have been reported as 5-30 $\mu \mathrm{m}$ long with a thickness of 0.1-2 $\mu \mathrm{m}$ while HABs are typically longer and thicker reaching up to $100 \mu \mathrm{m}$ and $10 \mu \mathrm{m}$ respectively in the circumferential cross section [5, 17, 26, 27, 28]. Fu et al. [22] recently measured the span of LABs in axial cross-section and showed that the average span of the LABs was $45 \mu \mathrm{m}$. Based on the reported dimensions of LABs and HABs observed in the axial and circumferential directions, WEBs have been suggested to be parallel thin plates. A recent study has attempted to model their structures in three dimensions [29], however detailed analysis is required to refine the model to represent the experimental observations of LABs and HABs e.g. in their thickness and length. A schematic of the 3D model of the WEBs proposed in literature is shown in Figure 1 with the terms used in this paper to refer to the band dimensions in 3D. 


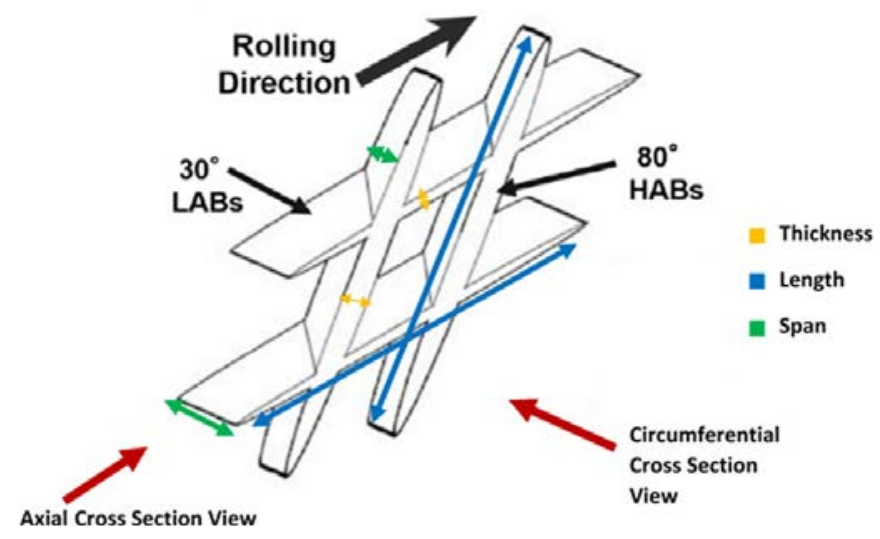

Figure 1: Schematic showing the 3D WEB network proposed from literature. The terms length, thickness and span used to refer to the dimensions of the LAB and HAB is shown for clarity. Adapted from [36].

Similar to DER, WEBs have been suggested to form in the region of maximum shear stress which varies depending on geometry and loading conditions $[14,17]$. However, majority studies were conducted under a single contact pressure. For example, Fu [21] concluded a DER orthogonal shear stress threshold by comparing DER formation over $1.2 \times 10^{7}$ and $1.2 \times 10^{8}$ cycles under $3.2 \mathrm{GPa}$ with the stress distribution. Table 1 summarises the locations of WEBs in RCF found inner rings reported in literature demonstrating discrepancies from different studies $[17,15,5]$. More research is thus required to study the evolution of the features throughout bearing life to understand their growth pattern and stress threshold if exists. Conflict conclusions have also been reported in literature regarding which stress component is responsible for the formation of which feature, e.g. some reported the formation of DER and WEBs to be driven by the principal shear stress [2, 8, 16, 27, 30] while others suggested it is due to the orthogonal shear stress [7, 15, 20] or von Mises stress [31]. A number of hypotheses have been proposed in literature explaining the formation mechanism of WEBs and their distinctive orientations based on combinations of the shear stresses acting on bearings, residual stresses and plastic strains present in the material [12, 32, 33, 34, 35]. Nonetheless, all hypotheses are seen to present some limitations and inconsistencies when compared to experimental investigations as discussed by the authors of this paper in [36].

A recent study has shown that WEBs consist of three components, i.e. globular/cellular ferrite grains, elongated ferrite grains and lenticular carbides (carbon-rich areas) [17], where the lenticular carbides form adjacent to the ferrite grains, also shown in [5, 7]. This is in agreement with previous studies in $[15,37,38]$ who proposed that the lenticular carbides form by the plastic deformation of the primary spheroidised carbides or carbide dissolution 
within the newly formed ferrite grain of the LAB. However this has been debated in $[16,17,21]$ that those two are independent of each other given the larger size of the lenticular carbides and that they are chromium depleted whereas the primary carbides are chromium rich. Fu et. al. [7, 39, 40] and Kang et. al. [30] proposed that the formation of $\mathrm{LAB}$ is due to dislocation- assisted carbon migration from the newly formed ferrite grains of the LABs to the edges leading to the formation and thickening of the lenticular carbides, however no consideration was provided on the formation of HABs [7, 30, 39, 40]. Polonsky et al. [32] suggested that HABs form due to gradual dissolution of the lenticular carbides which is maximised at an angle $45^{\circ}$ to the LABs which correlates with the HAB inclination angle observed experimentally. This was calculated by approximating the cyclic plastic behavior of WEB ferrite using the linear kinematic hardening law and neglecting out-of-plane plastic deformation for carbon outflow. However, there is a lack of experimental evidence showing links between LABs and HABs in bearings. There have been various models attempting to explain the development of DER and WEBs [12, 21, 38, 32] based on maximum shear stresses and carbon redistribution in the microstructure. However all models have shown some limitations.

Table 1: Depth boundaries of WEBs in bearing inner ring subsurface reported in literature

\begin{tabular}{ccccc}
\hline Reference & $\begin{array}{c}\text { Contact pressure } \\
(\mathbf{G P a})\end{array}$ & $\begin{array}{c}\text { Upper and lower } \\
\text { boundaries }(\boldsymbol{\mu m})\end{array}$ & Stress Cycles & Bearing type \\
\hline Swahn et al. [5] & 3.7 & $150-450$ & $1 \times 10^{9}$ & 6309 DGBB \\
\hline Fu et al. [22] & 3.3 & $180-470$ & $2 \times 10^{9}$ & 6309 DGBB \\
\hline Martin et al. [16] & 3.3 & $180-710$ & $2 \times 10^{9}$ & 6309 DGBB \\
\hline Smelova et al. [17] & 2.9 & $50-225$ & $3 \times 10^{9}$ & $7205 b$ ACBB \\
\hline
\end{tabular}

Despite significant amount of research over decades, the exact mechanisms and reasoning behind DER, LAB and HAB initiation and formation as well as the transition between them are not fully understood. The influence of material cleanliness, bearing operating conditions (load, speed, temperature and lubrication) on their formation is not fully defined either. This paper presents the results from a systematic study of DERs and WEBs (LABs and $\mathrm{HABs}$ ) in angular contact ball bearings (ACBBs) providing new experimental evidence of their characteristics and evolution mechanisms through optical microscopy based on samples from various stages across the bearing life. 


\section{Methodology}

All specimens analysed in this study were cut from through-hardened SAE 52100 (100Cr6) angular contact ball bearing inner rings subject to a heat treatment of austenitisation at $850^{\circ} \mathrm{C}$ for 30 minutes followed by quenching and tempering at $220^{\circ} \mathrm{C}$ for 2 hours. Two different grades of steel cleanliness are also examined in this study referred to as high cleanliness (HC) and very high cleanliness (VHC). The composition of the two steels is shown in Table 2. The cleanliness ratings of the steels are shown in Table 3 based on DIN 50602:1985 rating. The steels have a typical martensitic microstructure consisting of tempered martensite with homogeneously distributed primary $(\mathrm{Fe}, \mathrm{Cr})_{3} \mathrm{C}$ spherical carbides, tempered carbides and $<5 \%$ retained austenite.

Table 2: Chemical composition of high cleanliness (HC) and very high cleanliness (VHC) SAE 52100 samples

\begin{tabular}{|c|c|c|c|c|c|c|c|c|c|c|c|c|c|c|c|c|c|c|}
\hline & \multicolumn{14}{|c|}{ Weight \% } & \multicolumn{4}{|c|}{$\begin{array}{c}\text { Parts per } \\
\text { Million (ppm) }\end{array}$} \\
\hline & C & Si & Mn & $\mathbf{P}$ & S & $\mathrm{Cr}$ & $\mathbf{N i}$ & Mo & $\mathbf{C u}$ & $\mathbf{V}$ & Al & As & Sn & $\mathbf{W}$ & $\mathbf{P b}$ & Sb & $\mathbf{T i}$ & $\mathbf{O}$ \\
\hline $52100 \mathrm{HC}$ & 0.93 & 0.3 & 0.34 & 0.01 & 0.004 & 1.49 & 0.02 & 0.005 & 0.066 & 0.004 & 0.003 & 0.002 & 0.009 & 0.007 & 10 & 20 & 10 & 12 \\
\hline $52100 \mathrm{VHC}$ & 0.95 & 0.3 & 0.28 & 0.01 & 0.001 & 1.5 & 0.12 & 0.03 & 0.112 & 0.004 & 0.025 & 0.006 & 0.009 & 0.007 & 5 & 18 & 13 & 4 \\
\hline
\end{tabular}

Table 3: Steel cleanliness evaluated according to DIN 50602:1985 for the high cleanliness (HC) and very high cleanliness (VHC) AISI 52100 bearing steels

High Cleanliness (HC) Samples

\begin{tabular}{llllll}
\hline & K0 & K1 & K2 & K3 & K4 \\
\cline { 2 - 5 } Stretched Sulphides (SS) & 37.6 & 22.9 & 11 & 3.4 & 0 \\
\hline Dispersed Oxides (OA) & 0.17 & 0.1 & 0.08 & 0 & 0 \\
\hline Stretched Oxides (OS) & 0.06 & 0 & 0 & 0 & 0 \\
\hline Globular Oxides (OG) & 1.18 & 0.31 & 0.08 & 0 & 0 \\
\hline All Oxides Kx (O) & 1.41 & 0.42 & 0.17 & 0 & 0 \\
\hline \multicolumn{1}{c}{ Total Kx (O+S) } & 39.1 & 23.3 & 11.2 & 3.4 & 0 \\
\hline \multicolumn{1}{c}{ Very High Cleanliness } & (VHC) & Samples & & \\
\cline { 2 - 5 } & $\mathbf{K 0}$ & $\mathbf{K 1}$ & $\mathbf{K 2}$ & $\mathbf{K 3}$ & $\mathbf{K 4}$ \\
\hline Stretched Sulphides (SS) & 0 & 0 & 0 & 0 & 0 \\
\hline Dispersed Oxides (OA) & 0.4 & 0.2 & 0 & 0 & 0 \\
\hline Stretched Oxides (OS) & 0.9 & 0.4 & 0.3 & 0.1 & 0 \\
\hline Globular Oxides (OG) & 2.9 & 0.7 & 0 & 0 & 0 \\
\hline All Oxides Kx (O) & 4.2 & 1.3 & 0.3 & 0.1 & 0 \\
\hline Total Kx (O+S) & 4.2 & 1.3 & 0.3 & 0.1 & 0 \\
\hline
\end{tabular}




\subsection{RCF Testing}

Detailed analysis of DER, LAB and HAB formed in the inner ring of RCF tested ACBBs on an industry L17 test rig under two different contact pressures of 2.9 GPa and 3.5 GPa over a range of pre-determined stress cycles, ranging from 8 million to 4141 million cycles at a speed of 12,000 rpm. The bearing was lubricated using an ISO VG 68 oil while the temperature of the lubricant was maintained at $80^{\circ} \mathrm{C}$. Bearings at two different grades of steel cleanliness have been tested to evaluate the influence of steel cleanliness on DER/WEBs formation. It should be noted that none of the samples analysed in this paper demonstrated any surface damage. A summary of the test conditions of the samples investigated is given in Table 4, where a unique ID is given to the bearings accordingly for easy tracking.

Table 4: A summary of the ACBB samples investigated. Bearing ID is given for each sample based on their pressure-steel cleanliness-stress cycle information.

\begin{tabular}{|c|c|c|c|c|}
\hline Bearing ID & $\begin{array}{c}\text { Contact Pressure } \\
\text { (GPa) }\end{array}$ & $\begin{array}{l}\text { AISI52100 } \\
\text { Steel } \\
\text { Cleanliness } \\
\text { Grade } \\
\end{array}$ & $\begin{array}{c}\text { Stress Cycles } \\
\text { for Inner Ring } \\
\text { (Million) }\end{array}$ & $\begin{array}{c}\text { Test Duration } \\
\text { (hrs) }\end{array}$ \\
\hline 3.5-HC-151 & 3.5 & High & 151 & 27.6 \\
\hline 3.5-HC-288 & 3.5 & High & 288 & 52.6 \\
\hline 3.5-HC-447 & 3.5 & High & 447 & 81.7 \\
\hline 3.5-HC-679 & 3.5 & High & 679 & 124.3 \\
\hline 3.5-HC-885 & 3.5 & High & 885 & 162 \\
\hline 2.9-HC-591 & 2.9 & High & 591 & 107.6 \\
\hline 2.9-HC-1116 & 2.9 & High & 1116 & 203.2 \\
\hline 2.9-HC-1689 & 2.9 & High & 1689 & 307.6 \\
\hline 2.9-HC-2341 & 2.9 & High & 2341 & 426.3 \\
\hline 2.9-HC-3016 & 2.9 & High & 3016 & 549.3 \\
\hline 2.9-VHC-8 & 2.9 & Very High & 8 & 1.5 \\
\hline 2.9-VHC-109 & 2.9 & Very High & 109 & 19.8 \\
\hline 2.9-VHC-1337 & 2.9 & Very High & 1337 & 243.5 \\
\hline 2.9-VHC-2961 & 2.9 & Very High & 2961 & 539.4 \\
\hline 2.9-VHC-4141 & 2.9 & Very High & 4141 & 754.3 \\
\hline
\end{tabular}

\subsection{Microstructural characterisation methodology}

Standard metallography preparations have been conducted on all RCF tested ACBBs obtaining samples for 
feature examination. Samples from the tested bearings have been mechanically polished successively using $6 \mu \mathrm{m}$, $3 \mu \mathrm{m}, 1 \mu \mathrm{m}$ and $0.25 \mu \mathrm{m}$ diamond suspensions. After polishing, samples were etched in $2 \%$ Nital for $1-3$ seconds to observe the DER and WEB features under LOM (Olympus BX51). One of the challenges is to differentiate coexisted LABs and HABs in one sample when the two features overlap. It has been found that using a polarised light LABs and HABs can be effectively differentiated under LOM (see examples shown in Figure 2), thus it has been used in this study to identify and quantify the two features especially when they overlap. The density of the features is quantified based on their contrast using the software ImageJ. A suitable threshold is applied to the optical images of LABs and HABs from each sample to highlight the area covered by each feature under x20 magnification optical image as shown in Figure $2 \mathrm{~b}$ and $2 \mathrm{~d}$. The $\%$ area is used to quantify the density of the LABs and HABs (black area in Figure 2b and 2d relative to the whole image). For each bearing two cuts at random locations across the inner ring circumference were analysed and three measurements of the LAB/HAB \% area in each cut were obtained. The average of the three measurements of both samples is used to represent the quantity of the two features in each bearing.
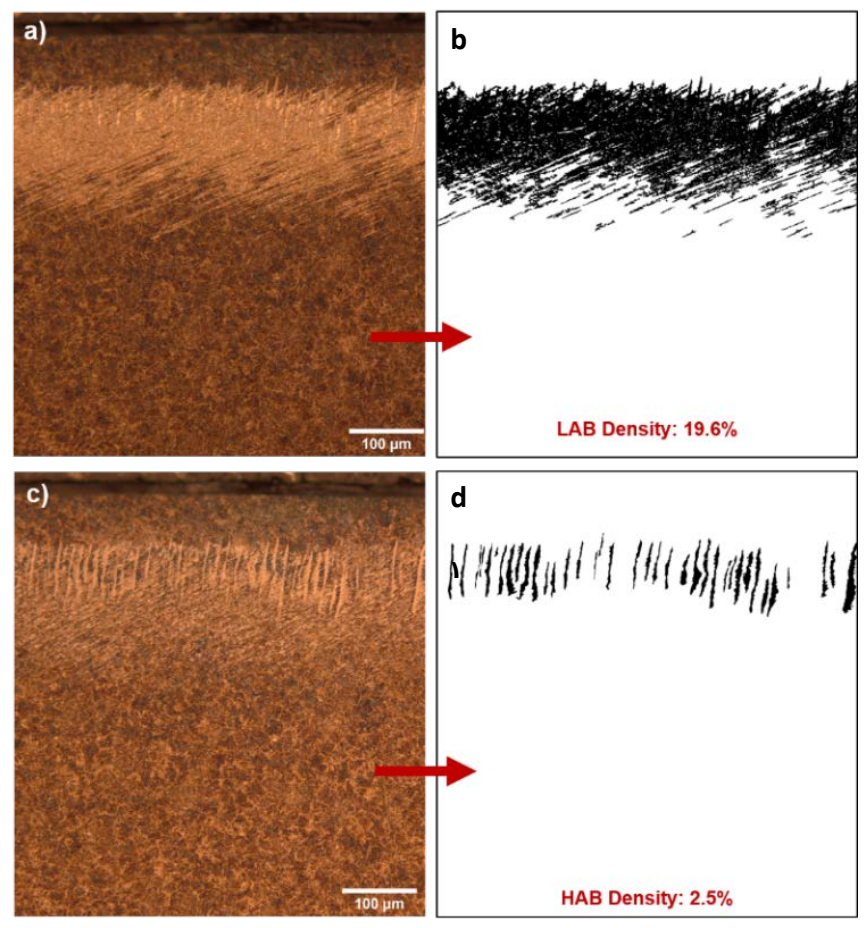

Figure 2: Optical images of bearing 2.9-VHC-4141 after polarizing light is applied to highlight a) LAB and c) $\mathrm{HAB}$ in an area on this bearing. b) and d) are images corresponding a) and c) respectively obtained using ImageJ, which is used to quantify the area covered by LABs and HABs. 


\subsection{Serial Sectioning}

Manual serial sectioning has been performed on bearing samples to investigate the 3D structure of LABs and HABs to verify the hypothesis given in literature. Sample 2.9-VHC-4141, where late stage LABs were found to have formed, was selected for this investigation, where serial sectioning at $5 \mu \mathrm{m}$ intervals was conducted to obtain a stack of optical images. ImageJ and Avizo 9.3 were then used to construct a 3D model of the LABs and HABs. The surface removal rate was measured and controlled using Vickers micro-indentations. A total of 140 slices were obtained. An illustration of the serial sectioning is shown in Figure 3.
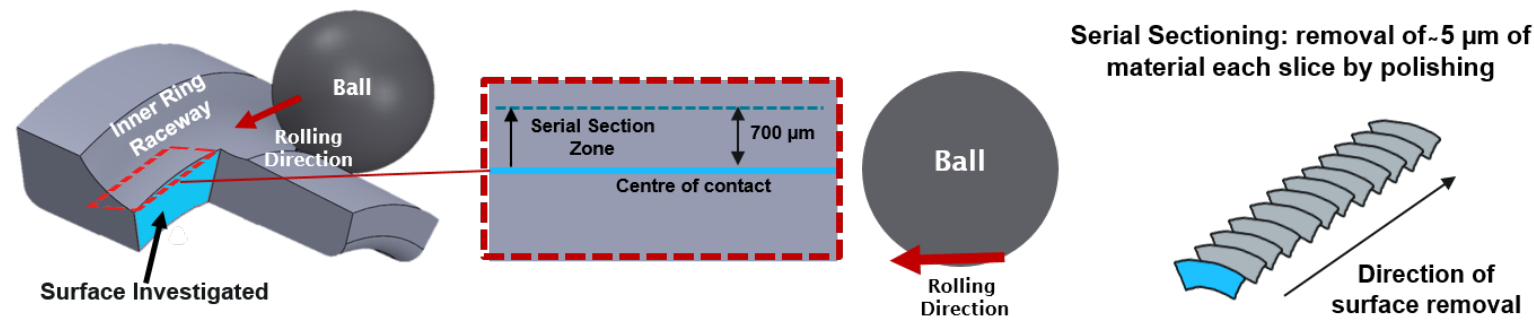

Figure 3: An illustration of the serial sectioning process on the circumferential cross section (blue area) of the inner ring for $\mathrm{LAB}$ and $\mathrm{HAB}$ mapping.

\section{Results}

\subsection{Characteristics of DERs and WEBs and their evolution}

To study the characteristics and their evolution of DER and WEBs under different conditions, the features formed in the bearings (from low to high stress cycles) have been examined and quantified using the procedure defined above. Figure 4 shows example LOM images of DER, LABs and HABs observed in both axial and circumferential cross sections of bearing inner rings from Test 2.9-HC-3016 and Test 2.9-VHC-4141, illustrating methods used to characterize the features especially their depth and boundaries. In the axial cross section (Figure 4b), the DER appears as a sickle shape with a width similar to the contact width between the inner ring raceway and ball, while the LABs appear as lines parallel to the raceway surface. HABs on the other hand appear in the axial cross section as white blocks (confirmed through 3D modelling of the HABs). In the circumferential cross section (Figure 4c), the DER appears as a dark band parallel to the raceway while the WEBs are inclined to the surface at approximately $30^{\circ}$ (LABs) and $80^{\circ}$ (HABs). 
The positions of the upper and lower boundaries as well as the center of the DER and WEBs formed in the HC samples under both contact pressures are shown in Figure 5. The positions of the features in the VHC steel at 2.9 GPa were found to be similar to those of the HC steel under 2.9 GPa, hence are not presented here. The DER measurements were conducted in the axial cross sections at the middle of the sickle shape as shown in Figure 5a where the depth of DER is at its deepest. All WEBs were measured in the circumferential cross sections to capture the features as demonstrated in Figure 5a (right).

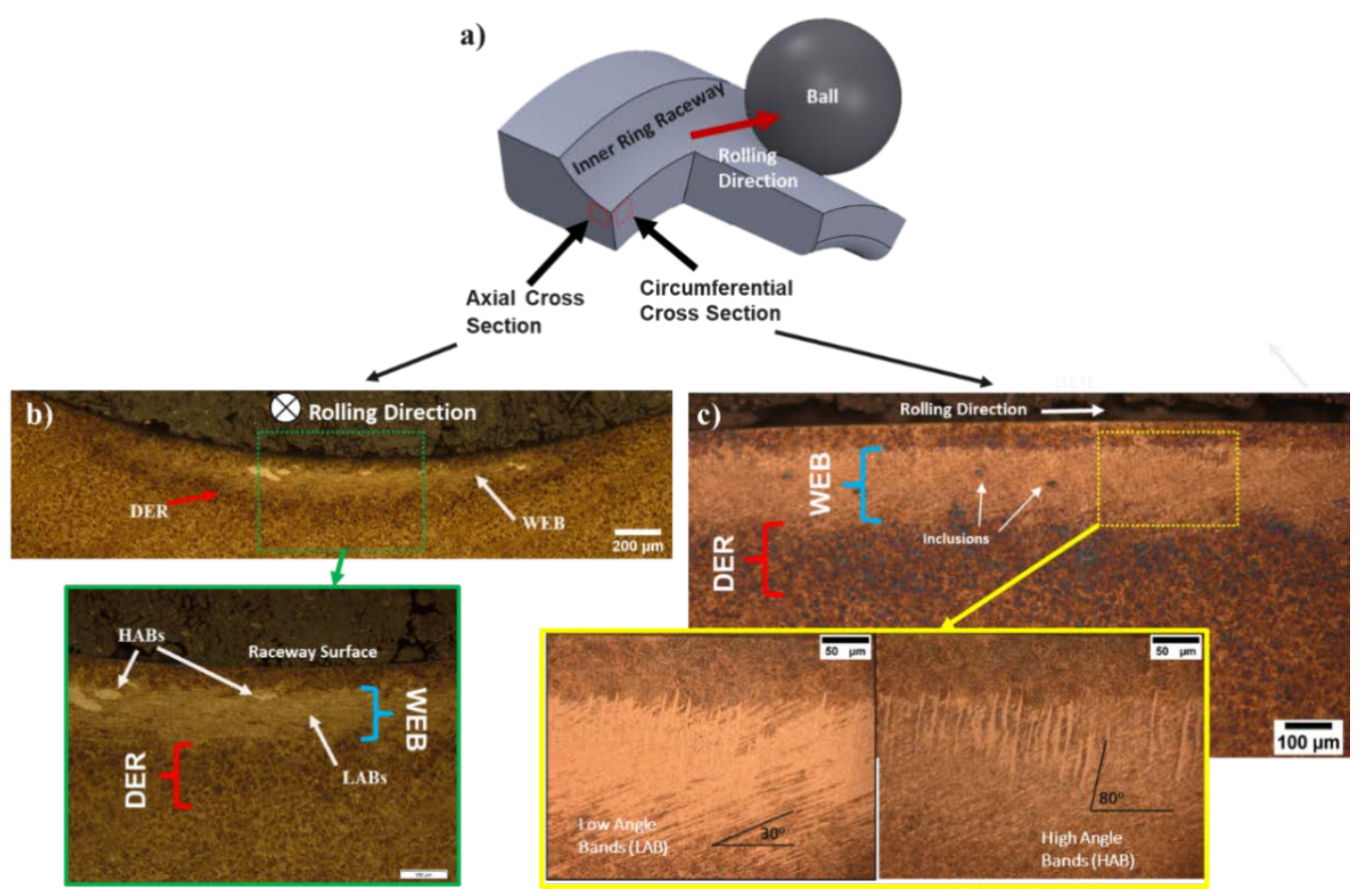

Figure 4: a) An illustration of the ACBB showing cutting directions performed on bearing inner ring. b) Fully developed DER and WEB features observed in axial section. c) DER and WEB features in a circumferential section of an inner ring with images at a higher magnification showing LABs and HABs in the same area where filters have been applied highlight the particular feature. Images from sample No. 2.9-HC-3016 and 2.9-VHC4141.

It can be seen that the depth of DER/WEB features increases with both stress cycles and contact pressure. The depth distributions of the corresponding von Mises stress, principle shear stress $\tau_{45}$ and orthogonal shear stress $\tau_{0}$ calculated based on Hertzian theory are shown on Figure 5 and it can be seen that the maximum von Mises and principal shear stress coincides with the mean depths WEBs under both contact pressure conditions while the DER appeared in deeper positions in the microstructure from the very stages. 
a)
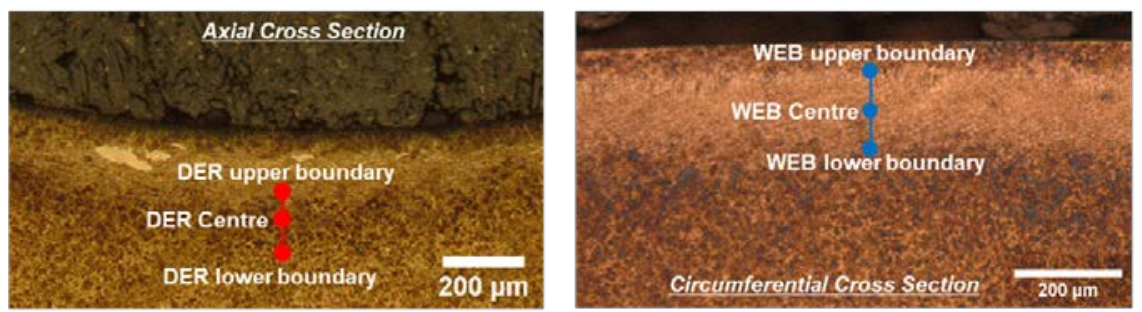

b)

\section{$2.9 \mathrm{GPa}$}
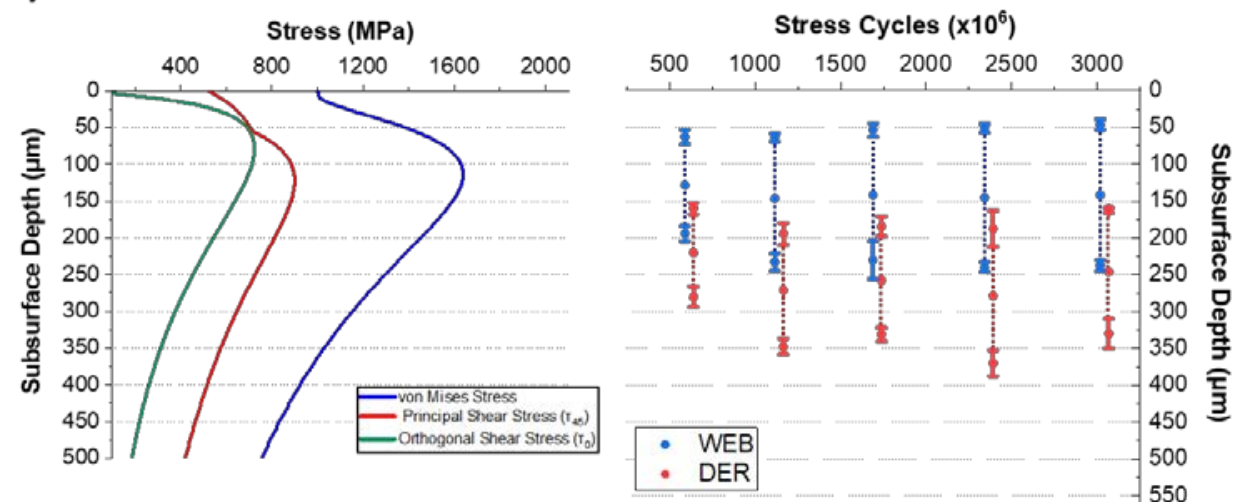

c)

\subsection{GPa}

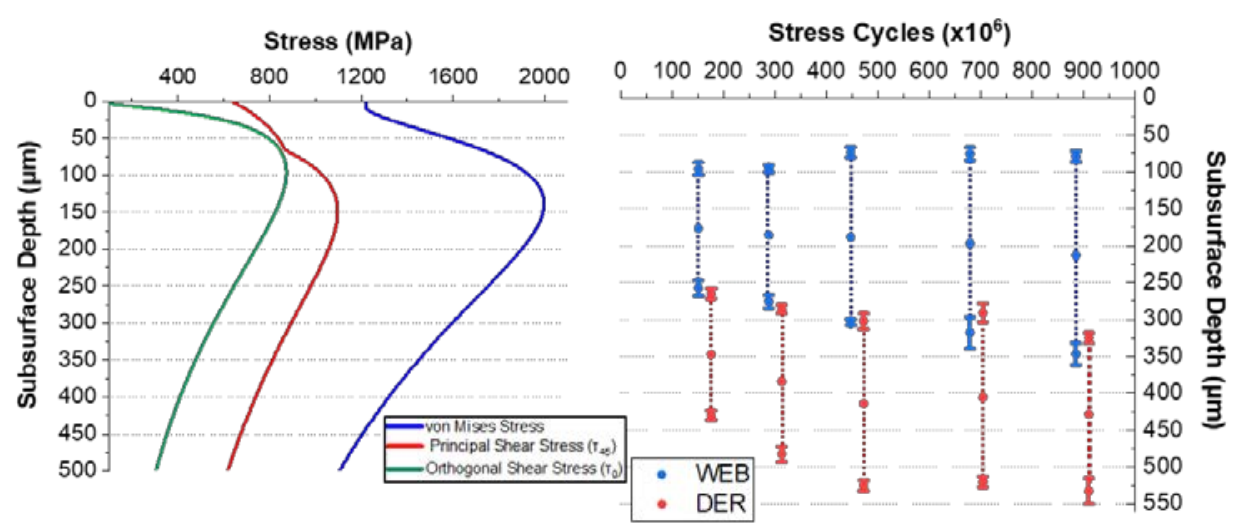

Figure 5: DER/WEB depth vs stress/load cycles for the HC steel bearings. a) Defined positions of the boundaries and centre depth of DER and WEBs measured at b) $2.9 \mathrm{GPa}$ and c) $3.5 \mathrm{GPa}$ compared to the corresponding von Mises stress, principal shear stress $\tau_{45}$ and orthogonal shear stress $\tau_{o}$ as shown in b) and c). It should be noted that the DER measurements are presented slightly shifted to the right for clarity.

It is also shown in Figure 5 that the increase of the contact pressure from 2.9 to 3.5 GPa has widened the spread (distance between upper and lower boundaries) for both features and increased the midpoint depth of DER and WEBs. Comparing the boundaries of WEBs, it can be seen in Figure 5b that after significant cycles (1500-2000 million) the boundaries of the WEBs reached a maximum (saturate). Figure $5 \mathrm{c}$ on the other hand, which is recorded under lower stress cycles, shows the lower boundary of the WEBs to increase (move deeper) at a faster rate 
compared to the upper boundary. This appears to coincide with the sharp/gradual reduction of stress (von Mises and $\tau 45$ ) from the maximum stress towards/away from the surface, which suggests the initiation of the WEBs is likely to have been in the region of the maximum von Mises or principal shear stress $\tau 45$. For the depth measurements, the depth was measured three times in two different cuts across the inner ring for each bearing giving a total of six measurements per bearing which have been averaged with standard deviation. Based on the WEB position in the samples, it is apparent that the shear stress in the subsurface has a major role in their formation, there appears to be avon Mises threshold in the order of $1300 \mathrm{MPa}$ at which the WEBs form.

On many occasion, WEBs and DER are found to be separated in this study which opposes to those reported in literature that WEBs form within DERs $[5,8,26,1]$. As the results above show that WEBs form in a shallower region compared to the DER. It might be argued that the dense WEB network formed has overlapped with preexisting DER, making it challenging to identify the true DER upper boundary and hence it is possible that only a segment of the DER has been observed in some cases (below the WEBs). The WEB boundaries in the samples also presented a distinctive boundary (both upper and lower) which made their distinction more obvious compared to the DER boundaries which was fading gradually into the parent microstructure leading to potential uncertainties in their true position.

\subsection{Density of LABs and HABs with Cycles}

LABs and HABs formed in same bearings have been quantified by applying appropriate thresholds to the polarized LOM images to quantify their densities as discussed section 2.2. The \% density of LAB/HAB is defined as the area covered by the LABs and HABs (black area in Figure 2b and d) within a fixed area of $825 \mu \mathrm{m}$ x 663 $\mu \mathrm{m}$ (x20 mag image size) as shown in Figure 2 . Hence, a \% density of LAB of $15 \%$ would suggest $15 \%$ of the captured image area consists of LABs.

The results have been summarized in Figure 6 where the densities of the LABs and HABs for the two steels under two contact pressures are shown. Initially the uniformity of the WEB network around the whole circumference of the bearing inner ring was investigated by taking four random cuts each from the inner ring of the bearings 2.9HC-3016 and 2.9-VHC- 4141. Comparing the results from multiple cuts of a bearing, the densities of LABs show a small variation of $\leq 9 \%$ while of HABs a variation of up to $30 \%$ around the inner rings have been observed. 
Thus, two random locations between the four locations on each bearing inner ring have been subsequently analysed and their average is used to represent the density of the features for the relevant test. For each of the two locations, the density measurement is applied three times to give a total of six measurements for each sample which is averaged and standard deviation is calculated.

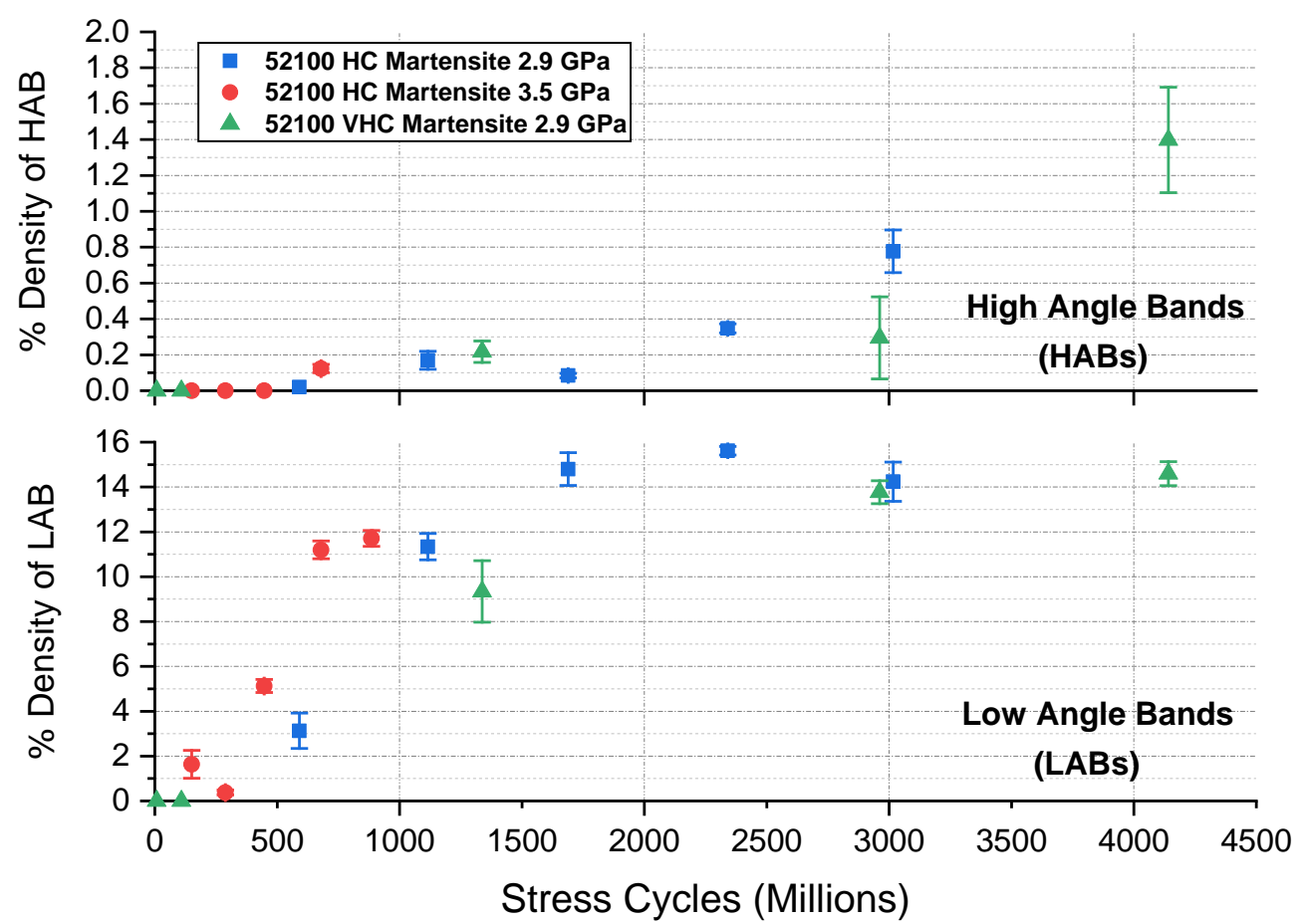

Figure 6: Normalised density of LAB (bottom) and HABs (top) vs cycles for HC steel under 2.9 GPa and 3.5 GPa and VHC steel under 2.9 GPa.

As shown in Figure 6, the densities of LABs and HABs over stress cycles have different trends for the three conditions. For the HC 2.9 GPa samples, LABs show a steady growth from the early stages until approximately 1700 million cycles when a saturated level has been reached. HABs were not observed until after 679 million cycles, similar to findings in literature [5]. A modest level of HABs is maintained until around 1700 million cycles when a sudden increase occurred, which coincides with the point where LABs achieve its saturation, after which the HABs start to grow rapidly. This suggests that the formation of HABs is provoked by the high density of LABs formed in the region. Comparing these with the other two cases, the development of the LABs initiated earlier in the bearing life under higher contact pressure (2.9 GPa vs $3.5 \mathrm{GPa}$ ) and the cleaner steel (VHC) appears to modestly impede the development of LABs over similar cycles (comparing 2.9-VHC-1337 and 2.9-HC-1116). This suggests non-metallic inclusions may have a role in the development of WEBs. 
The hypothesis that HABs formation is dependent on the density of the LAB network is also supported by the fact that the HABs are positioned towards the upper boundary of the pre-existing LABs which contains a denser network of LABs. It can also be seen that the cycle stage where HABs start forming at a rapid rate (Figure 6) also corresponds to the point where the upper boundary of the LABs saturates in Figure 5, i.e. when they are fully developed. The early stage of HABs show the features as conglomerates whereas they become more uniformly distributed at later stages as shown in the lower optical images in Figure 7. At earlier stages of the LABs, prior to their saturation point between 1500-2000 million cycles, the distribution of LABs is not uniform as some local regions appear more dense than others as highlighted by the red circles in Figure 7a which also correlates with the position of early stage HABs. Beyond the saturation point of the LABs as shown in Figure 7b, the LABs become denser and uniformly distributed; hence, the HABs appear to be more uniformed, confirming that a dense LAB region is a prerequisite for HAB development.
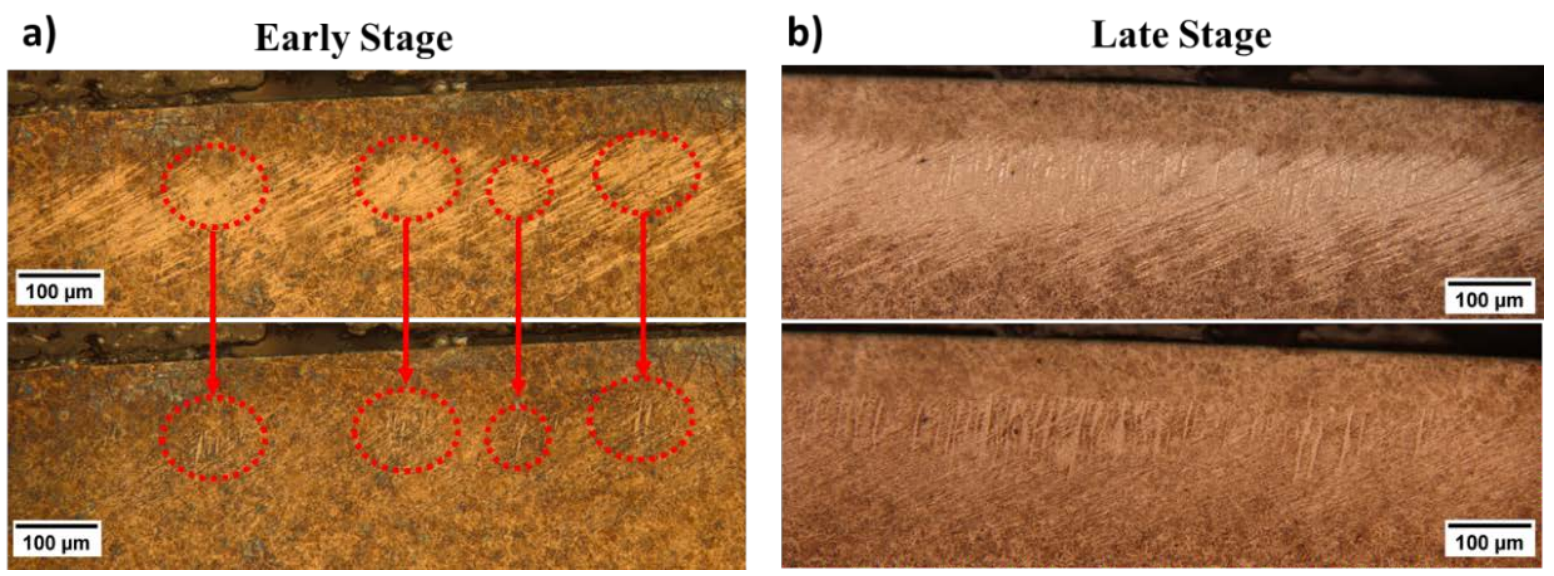

Figure 7: Optical image of LABs (top) and HABs (bottom) from samples a) 2.9-HC-1116 and b) 2.9-VHC4141. The dense LABs regions highlighted in red in a) correlates with the early development of HABs.

Figure 8 presents statistical data on the dimensions of HABs recorded in each sample under 2.9 GPa based on the terminologies highlighted in Figure 1. All measurements were taken from circumferential cross section cuts of the samples. HABs can be seen growing in both thickness (Figure 8a) and length (Figure 8b) throughout the bearing life while the span of the HABs is investigated through serial sectioning in the next section. 

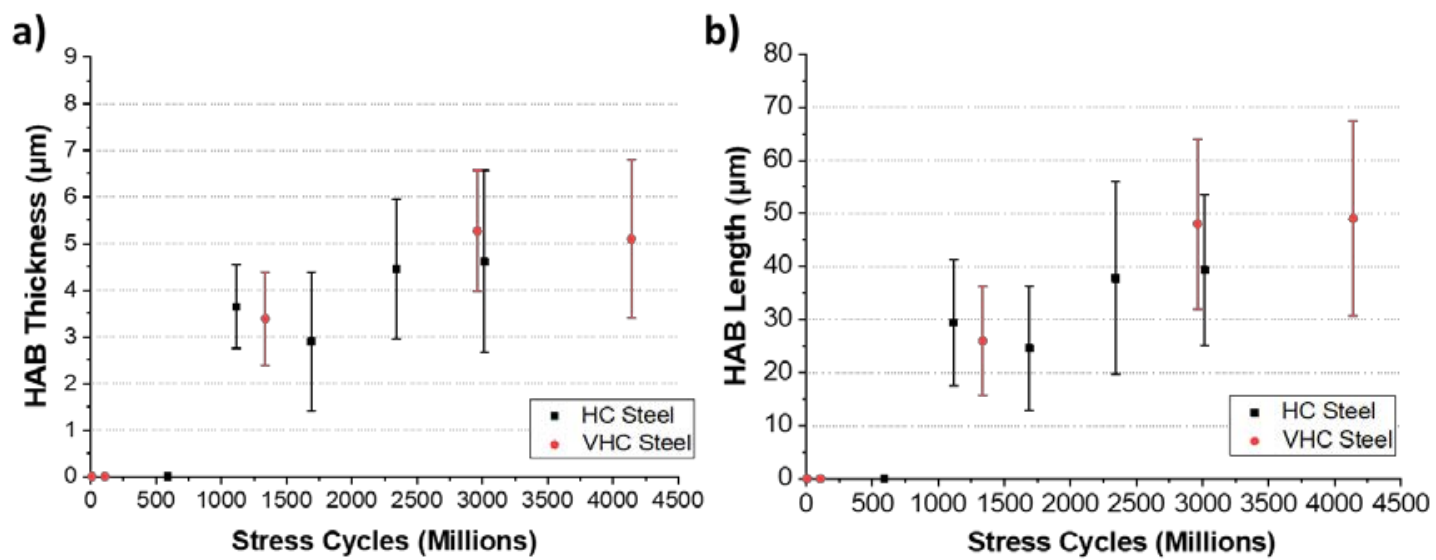

Figure 8: Statistical results with standard deviation on the a) thickness and b) length of HABs from HC and VHC steel bearings under various stress cycles under $2.9 \mathrm{GPa}$.

Further investigation of the $\mathrm{LAB}$ network is carried out by measuring the LAB density with respect to the depth below the raceway across different stages of the bearing life. This is achieved by marking slices with a depth of $10 \mu \mathrm{m}$ across the LAB threshold image (see illustration of the procedure shown in Figure 9). The LABs density distributions over the depth from the bearing surface for four of the tests under 2.9 GPa are shown in Figure 10. It is clearly shown that across all stages of development, from early to late stages, the density of the LAB correlates well with the distribution of the von Mises and principal shear stress. The early stage of LAB (Figure 10b) shows a sharp peak coinciding with the position of maximum stress which becomes broader at later stages but the position of the maximum LAB density coincides with the von Mises and principal shear stress (maximum at $120 \mu \mathrm{m}$ and $115 \mu \mathrm{m}$ respectively) across the bearing life rather than the orthogonal shear stress (maximum at $80 \mu \mathrm{m}$ ). This suggests the point of the highest subsurface stress (principal shear) is likely the origin of the LAB features. The upper boundary of the LABs is also seen to increase in density at a faster rate compared to the lower boundary i.e. reaches saturation quicker than the lower boundary. By comparing the density of the LABs with the position of HABs, an obvious correlation is observed in Figure 10c,d\&e where the HAB is located in the region of the highest LAB density. 


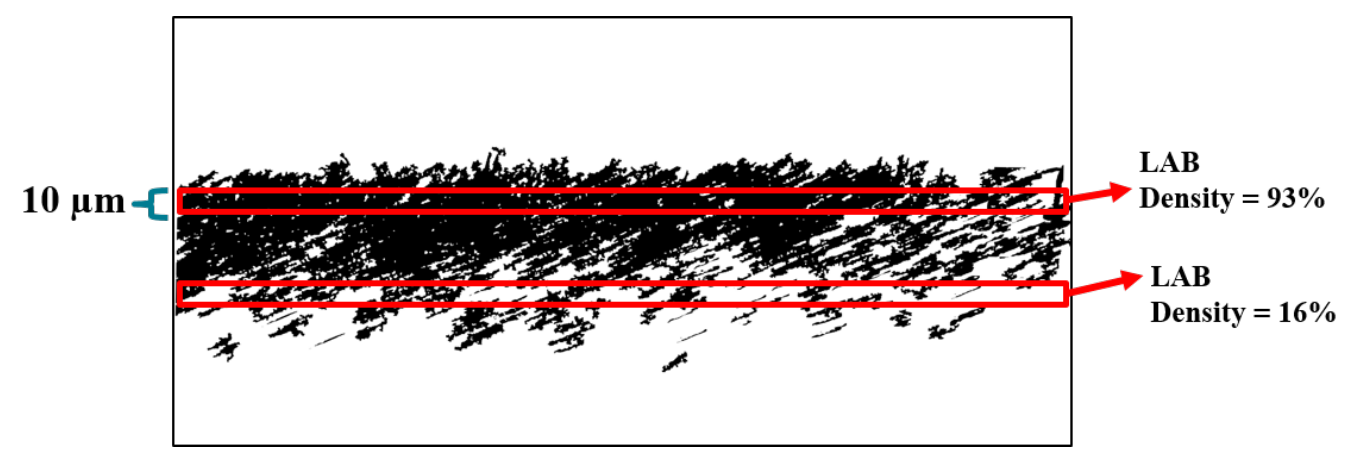

Figure 9: Illustration of the method used to determine the LAB density variation with depth. Drawing of slice is not drawn to scale for clarity.
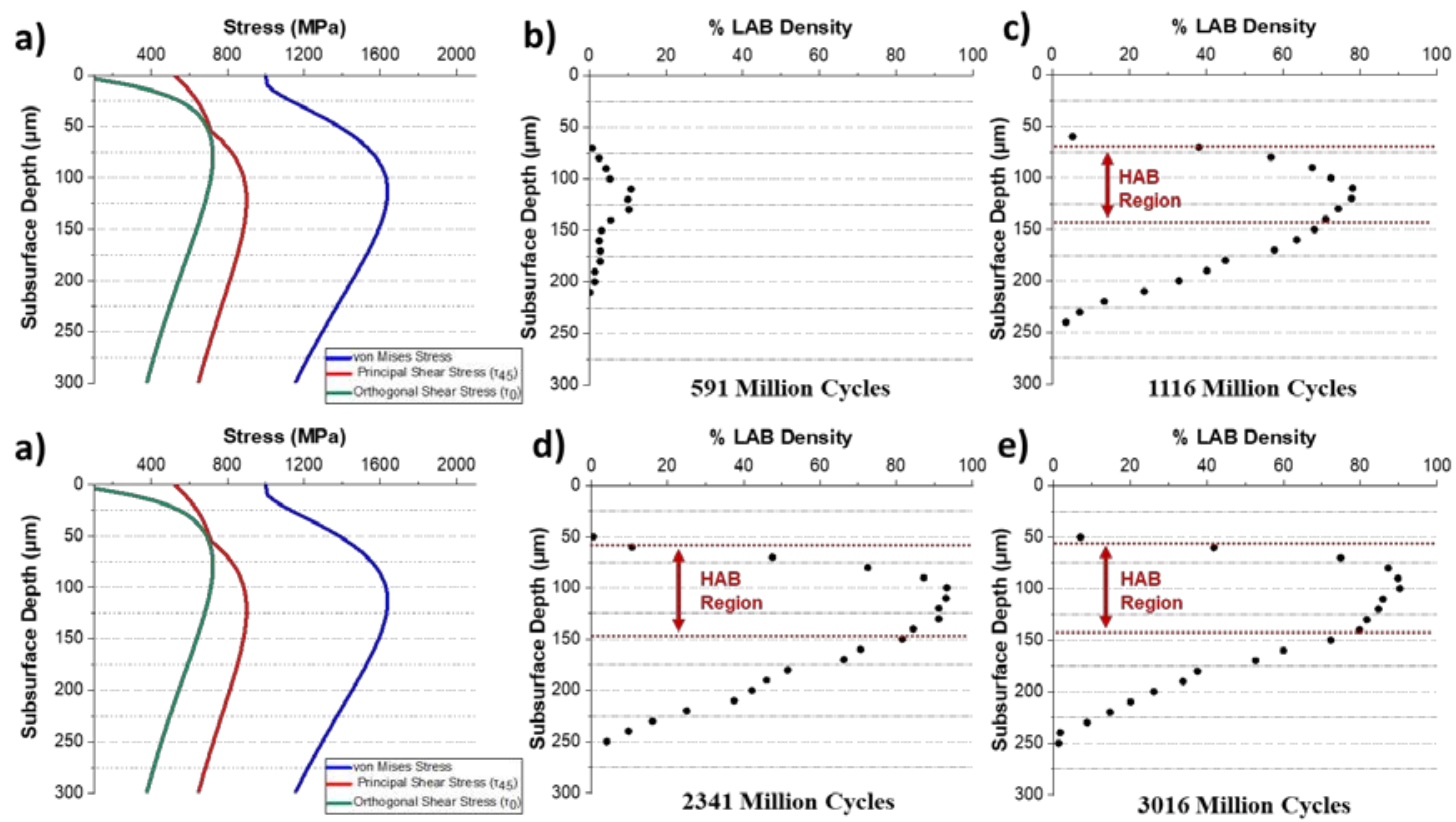

Figure 10: a) Subsurface stress distribution of von Mises stress $\left(\sigma_{\mathrm{vM}}\right)$ principal shear stress $\left(\tau_{45}\right)$ and orthogonal shear stress $\left(\tau_{0}\right)$ under a contact pressure of $2.9 \mathrm{GPa}$. LAB density distribution across the depth is shown from sample b) 2.9-HC-591 c) 2.9-HC-1116 d) 2.9-HC-2341 e) 2.9-HC-3016. Red lines in c-e represent the upper and lower boundary of HABs found in the corresponding sample.

\subsection{Serial Sectioning of WEBs}

Manual serial sectioning performed on the WEBs was used to construct a 3D model of the WEB network consisting of LABs and HABs. The model of the WEB network can be seen in Figure 11 where an orthogonal slice cut is performed in the xy plane ( Figure 11b) and yz plane ( Figure 11c) based on the directions indicated in Figure 11a. It can be seen in Figure 11b that the structure consists of dense LABs together with a few scattered HABs. When cutting the model with an orthogonal slice in the yz plane (Figure 11c) it is interesting to observe the LABs 
as thin parallel lines similar to that reported inliterature in the axial cross section while the HABs appear as dense blocks similar to that observed in Figure 4b which has not been shown in literature.
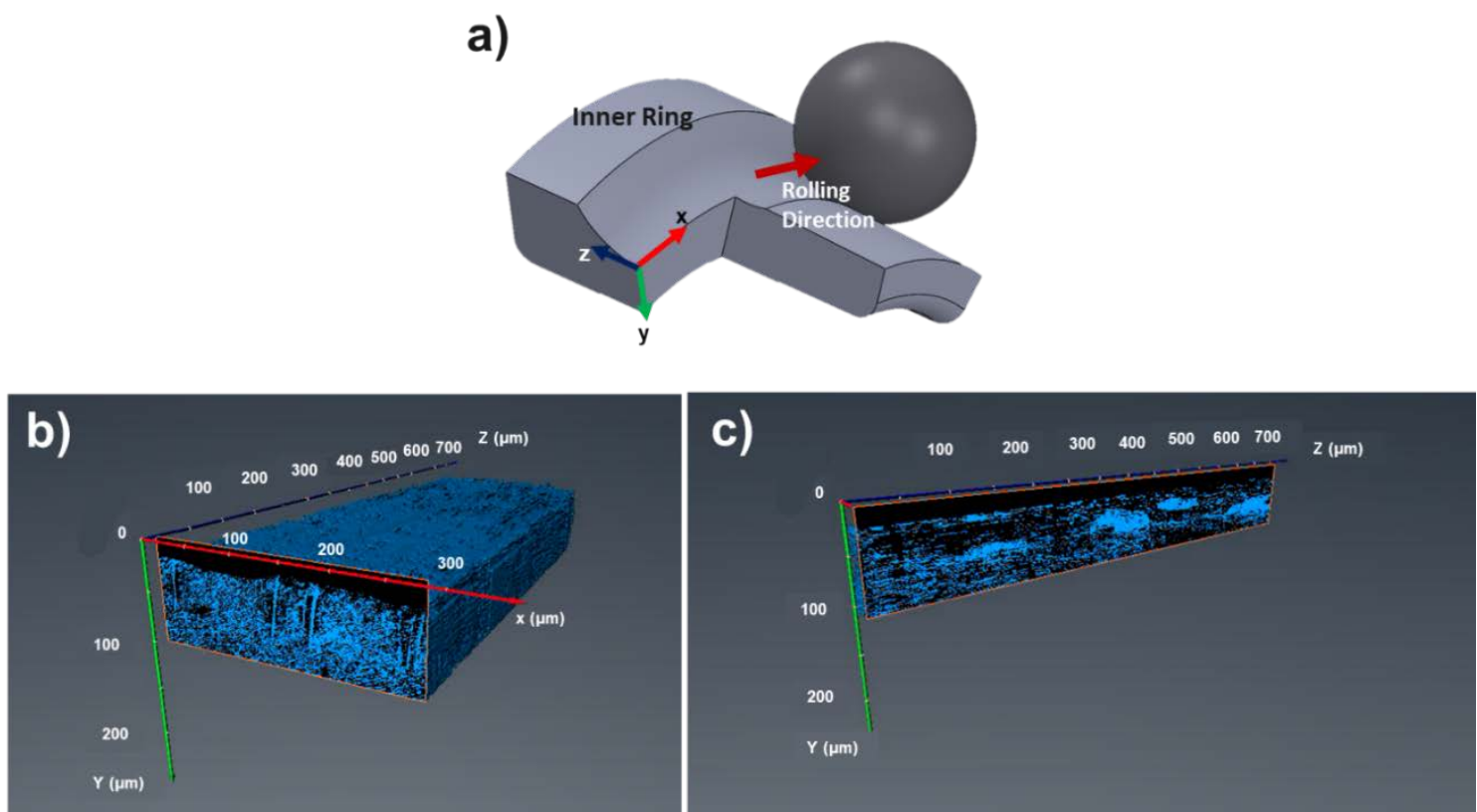

Figure 11: a) defined planes of the 3D model relative to the bearing geometry were xy plane corresponds to circumferential cross section and yz plane corresponds to axial cross section. b) A WEB 3D model with an orthogonal cut in the xy plane. c) A WEB 3D model with orthogonal cut in yz plane. See video 11 for serial orthogonal cuts of WEB 3D model in yz plane.

Further segmentation has been carried out on the WEB model highlighting individual HABs within the WEB network as demonstrated in Figure 12. The span of these features as shown in Figure 12b ranges from $25 \mu \mathrm{m}$ to over $575 \mu \mathrm{m}$ which corresponds to over a third of the half contact width b (in z direction). Throughout the HAB span, it appears to grow in both thickness and length with statistical data on the 3D dimensions of the HABs reported in Table 4 compared to 3D LAB dimensions recorded from the same sample.

Table 5: 3D dimensions of HABs and LABs obtained from serial sectioning results in sample 2.9-VHC-4141.

\begin{tabular}{lcccccc}
\hline & \multicolumn{3}{c}{ HAB } & \multicolumn{3}{c}{ LAB } \\
\cline { 2 - 7 } & Minimum & Average & Maximum & Minimum & Average & Maximum \\
\hline Length $(\mu \mathrm{m})$ & 13 & 60 & 90 & 12 & 52 & 118 \\
\hline Thickness $(\mu \mathrm{m})$ & 2 & 5.6 & 10 & 0.1 & 1.6 & 4.8 \\
\hline Span $(\mu \mathrm{m})$ & 25 & 160 & 575 & 9 & 44 & 105
\end{tabular}

Through serial sectioning, the edges of the HABs across its span could be observed to investigate the growth of the HAB features along the $\mathrm{z}$ direction in the $\mathrm{xy}$ plane of the $3 \mathrm{D}$ model. Overall there were three different characteristics / shapes observed when looking at the edge of the HABs demonstrated in Figure 13. One 
characteristic highlighted in Figure 13a is the HAB growing in thickness and length (towards and away from the surface) until it reaches its maximum size whereas the HAB in Figure 13b shows the HAB growing in length initially followed by thickness. It is interesting that some HABs, such as that demonstrated in Figure 13c, show the HAB initially consisting of separate small white etching matter from the LAB (orange arrow) joined together eventually to form a single HAB (red arrow) that later grows in length and thickness similar to others.
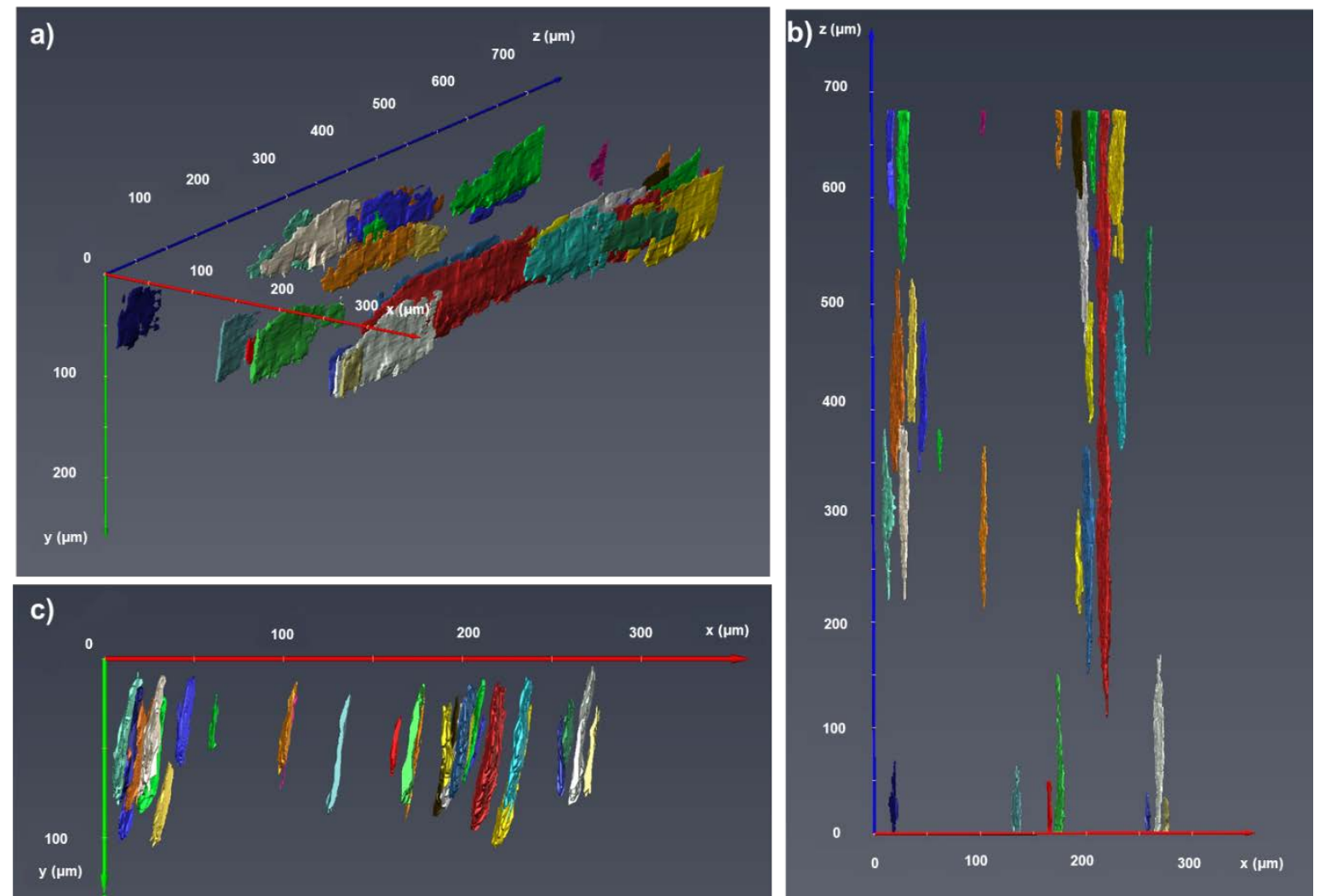

Figure 12: a) 3D model of multiple HABs within the WEB network in Figure 11. b) View of the 3D HAB model in the xz plane. c) View of the 3D HAB model in the xy plane. See video 12 for 360 degrees rotation of the 3D model.

The 3D model of the HABs was modified to show inclusions found within the volume of the WEB network(see Figure 14) to investigate their influence on the features in accordance with the results found in Figure 6. Given the sample investigated was from a VHC steel, onlyalimited number ofinclusions wereexpected tobefound. Althoughvarious inclusions were found in the WEB volume some small inclusions(less than $9 \mu$ mindiameter) were found at the edge and within HABs. Such inclusions are smaller than the k0 rating of DIN50602:1985 and hence are not accounted for in cleanliness assessments. While Figure 6 does suggest steel cleanliness may have a modest impact of WEB development, majority of HABs observed through serial sectioning appear to form independent of any inclusions 
surrounding them which suggests their influence on HAB is minimal where the existence of inclusions near/within HABs could be based on probabilities.

Nonetheless, it is obvious from Figure 15 the dimensions, density and depth of both DER and WEBs vary by shifting from the center of the contact area (Figure 15a) towards the edge (Figure 15b). It is apparent that the HAB density has reduced significantly while both WEB and DER features appear shallower compared to the features at the contact center. This observation is expected given the sickle shape shown in the axial cross section in Figure 4b.

a)
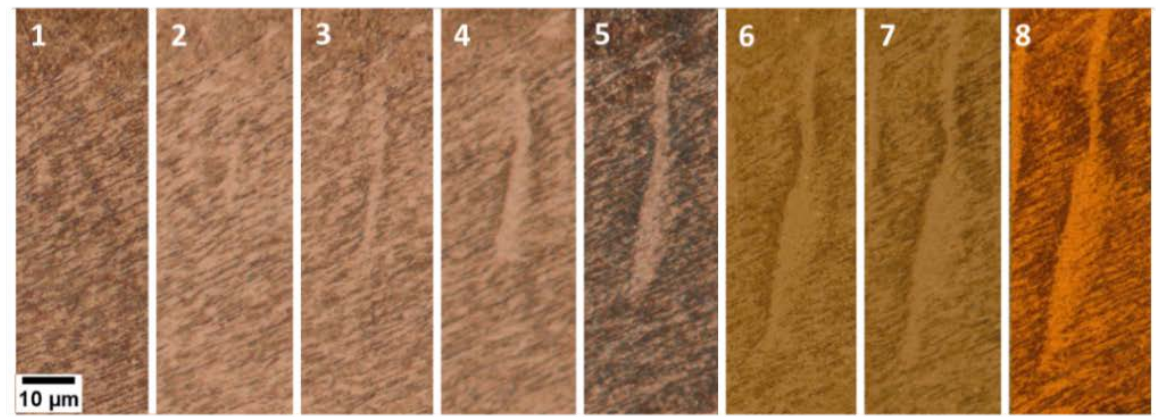

b) 1
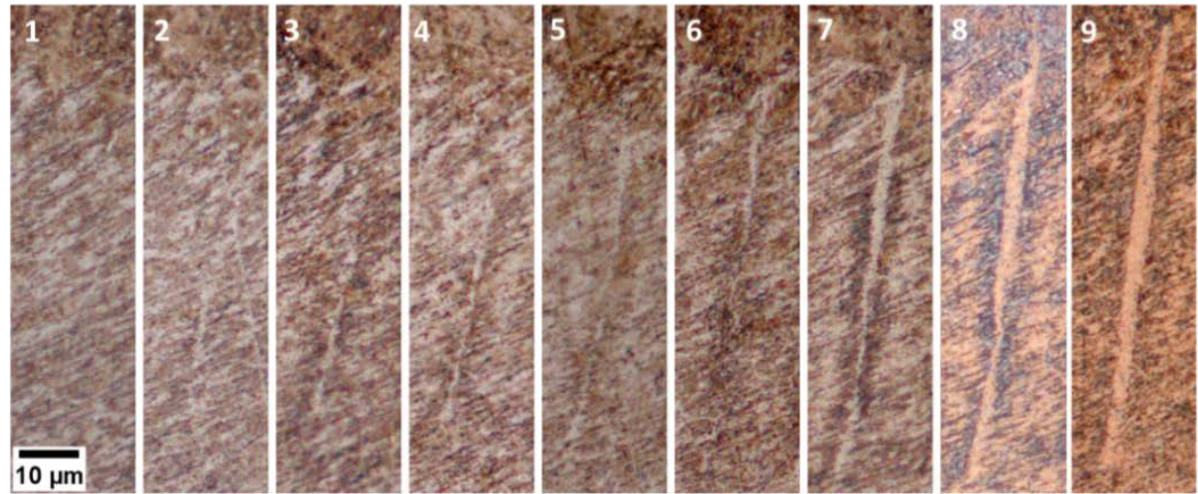

c) 1
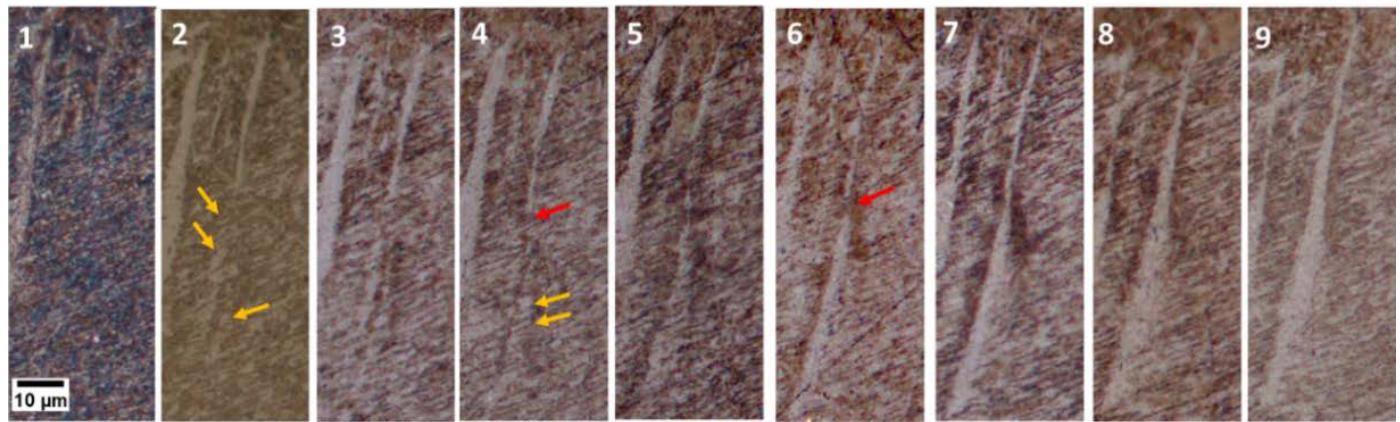

Figure 13: Growth of HAB from span edge to full development obtained from serial sectioning in xy plane showing a) HAB growing in thickness and length simultaneously b) HAB growing in length initially followed by thickness c) HABs forming from individual white etching matter from LABs which joins to form a single HAB. Numbers indicate number of slice with each being $10 \mu \mathrm{m}$ apart. Red arrows shows gaps between HABs prior to merging together while orange arrows show individual segment of white etched areas from LABs prior to becoming full HABs. 


\section{Discussion}

This study presents experimental characterisation of the progression of DER, LABs and HABs throughout the bearing life which is currently limited in literature. The depth of the WEBs in this study corresponds with the position of maximum equivalent stresses like principal shear stress in agreement with [8, 16, 22, 27, 30, 31]. As indicated from Figure 15, measurements of feature depth must be compared precisely at the center of the contact area since the slightest deviation from the center would affect measurement reliability, leading to disparity in measurements such as those found in literature regarding the stress distribution and feature position [7, 15]. While multiple findings have reported DER forming within the maximum shear stress region [7, 15, 20], the same conclusion could not be reached in this study.
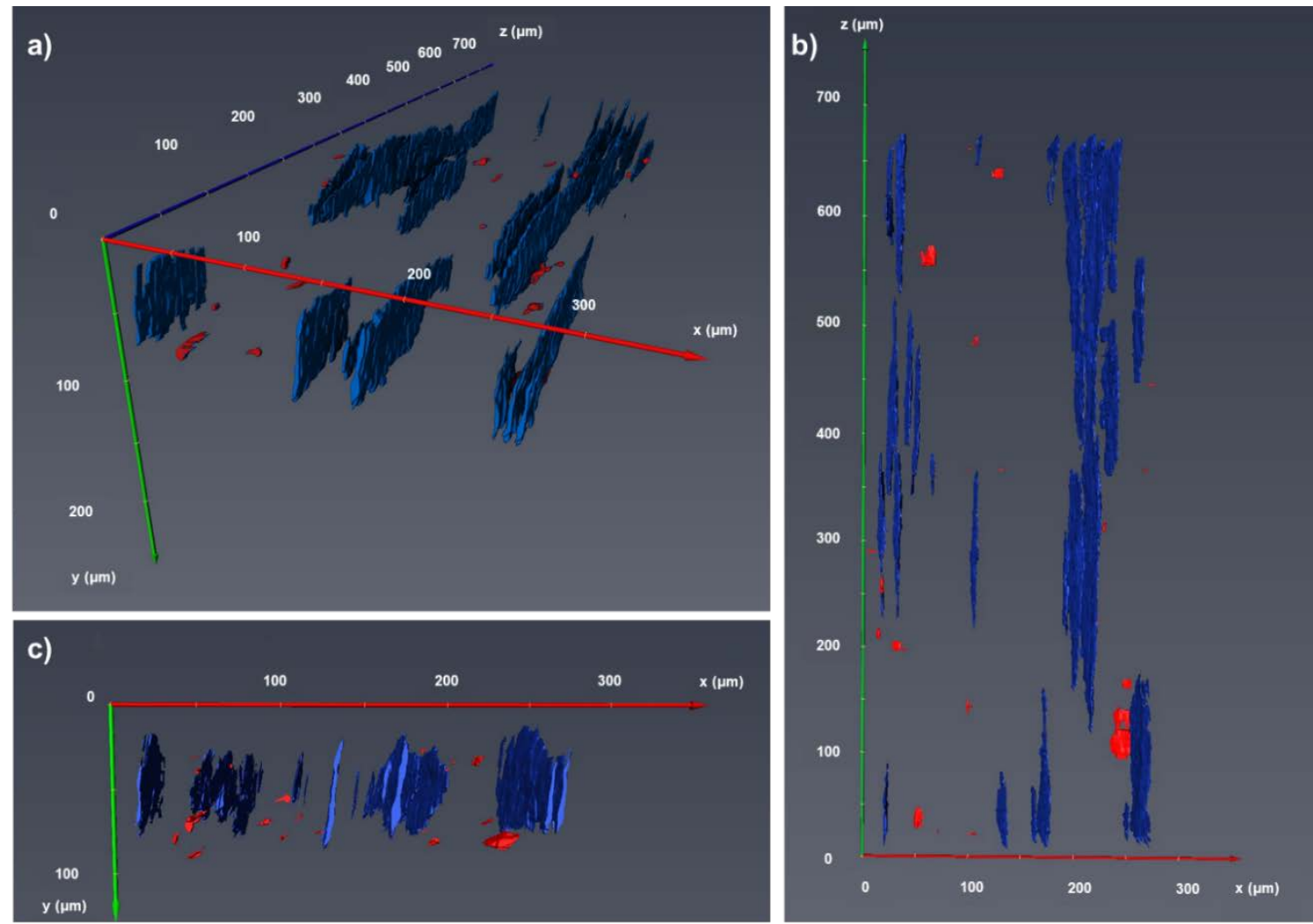

Figure 14: a) 3D model of HABs from Figure 12 compared to inclusion positions. b) View of the 3D $\mathrm{HAB}$ /inclusion model in the xz plane. c) View of the 3D HAB/inclusion model in the xy plane. See video 14 for 360 degrees rotation of the 3D model. 

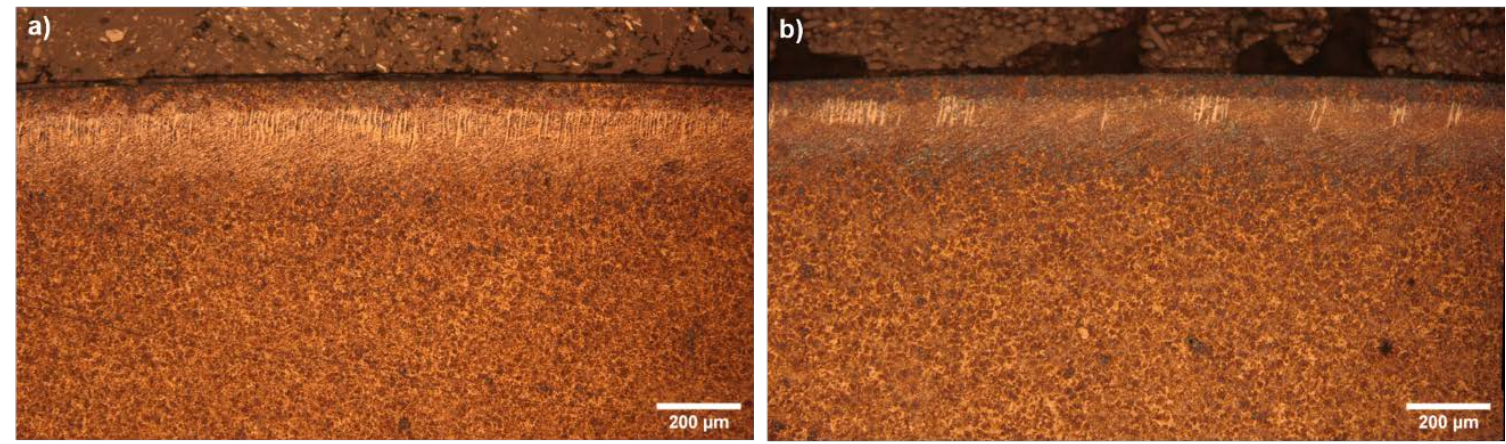

$550 \mu \mathrm{m}$ material removed

Figure 15: DER, LABs and HABs shown in the circumferential cross section on the bearing inner ring at a) centre of the contact area and b) $550 \mu \mathrm{m}$ away from the centre in direction perpendicular to rolling direction. Images obtained from serial sectioning.

\subsection{DER}

DER formation is suggested to be attributed to a stress-induced martensite decay process while the steel initial heat treatment has also been reported to impact its development. Steel samples tempered at $150-160{ }^{\circ} \mathrm{C}$ for 90 minutes have shown developed stages of DER after RCF testing [12, 15, 22]. Meanwhile higher tempering at 220$235^{\circ} \mathrm{C}$ for 4 hours did not show to have formed DER under similar RCF testing [12, 16, 22]. The heat treatment in this study is considered to be an intermediate between the extreme cases shown in literature (220 ${ }^{\circ} \mathrm{C}$ for 2 hours) and do show DER but not as pronounced as in other studies with lower tempering. It has been suggested that DER is formed due to dislocation assisted carbon migration towards tempered carbides in the microstructure [7, 30]. Given that higher tempering weakens the coherency of the tempered carbides to the matrix [12, 24], this may explain why higher tempering could hinder the formation of DER. However it is also possible that the process of carbon migration towards the carbides is more advanced during higher tempering arising from temperatureassisted diffusion and hence carbon migration during bearing operation of high tempered steel is not sufficient to induce DER formation.

It has been speculated that WEBs occur when a sample has a relatively low hardness in the order of $720 \mathrm{HV}$ or lower [22]. This theory would suggest for hard steels (low tempering), formation of DER would be required to soften the material to a level suitable for WEB development. Where for relatively soft steel (high tempering), WEB can form in the steel directly from the matrix given the hardness is already below the suggested threshold. In this study, DER has been identified at a position below the WEBs, i.e. not corresponding to the subsurface 
stress distribution. Rather than the WEBs forming at different positions to the DER in the bearing samples, it is believed the presence and growth of the WEBs (which occurs after DER formation) in the sample causes the DER in the WEB region to become no longer visible. Hence, as WEBs become more pronounced, the upper boundary of the DER fades away. Hence only the DER below/away from the dense WEBs can be observed in this study which may be only a segment of the full DER.

The hardness of the steel bearing sample in this study was examined through micro-indentation prior to RCF testing and found to be approximately $755 \mathrm{HV}$ which would agree in theory with the hardness threshold proposed for DER formation [22]. However, steel hardness is affected by various factors such as grain size, carbide size and distribution and heat treatment. Since the DER formation is governed by the carbon migration, excess carbon in solid solution should be a more suitable measure for determining a threshold for DER formation.

The shear stress analysis in the subsurface alone cannot explain the development of the DER where the consideration of residual stress development and heat treatment affecting the carbon migration is necessary to better understand the development of DER.

\subsection{WEBs}

LABs which have a preferred orientation ranging from $20-35^{\circ}$ show a growth pattern coinciding with the principal shear stress and von Mises stress rather than the orthogonal shear stress (see Figure 5 and Figure 10) from the earliest stage. It can also be assumed that a von Mises threshold in the order of $1300 \mathrm{MPa}$ appears as the saturation boundary of the WEBs in the late stages (Figure 5b) which confirms the features are stress induced. The threshold observed in this study is comparable to other thresholds proposed in literature corresponding to a principal shear stress in the order of $0.72 \mathrm{GPa}$ [16]. The data presented in Figure 5c shows the boundary limit lower than the proposed threshold due to the relatively lower stress cycles given the saturation level of WEBs is reached in Figure 5b between 1500 and 2000 million cycles. LABs grow initially at a steady state until a saturation level is observed between 1500 and 2000 million cycles (see Figure 6). Given WEBs are believed to be a manifestation of plastic deformation, it has been proposed by Fu et al. that the broadening on the WEB formation range should saturate when the principal shear stress equals the yield shear strength of approximately 0.74 GPa for SAE 52100 steel bearing [22]. This hypothesis coincides with the experimental results of this study (Figure 5). 
A recent model has attempted to model the growth of LABs with contact stress and cycles through the thickening of lenticular carbides forming adjacent to the $\operatorname{LAB}[22,39]$. Both the theoretical model proposed and the experimental LAB evolution results in this study (Figure 6) show LABs to grow at a steady rate initially until a saturation point is achieved. However as the model compares the growth of individual LABs until it becomes fully developed (reaches maximum thickness), this study shows the overall growth /density of the WEB network (LABs and HABs) and hence includes both the dimensions of WEBs and the number of bands formed. Both data show the LABs to grow under a linear relationship until the saturation level is reached while the rate at which it grows would vary depending on the material properties and operating conditions as shown in Figure 6 when comparing the features in the samples under 2.9 GPa vs 3.5 GPa.

Majority of WEB investigations in the literature is focused on LABs rather than HABs given the former develops earlier than the latter. Significant evidence in this study suggests the initiation of HABs stems from pre-existing LABs which is best shown in Figure 7. HABs initially appears in the material in conglomerates typically centered around the most dense $\mathrm{LAB}$ locations. As the LAB saturates, HABs become more evenly distributed resulting in a more uniform scatter of HABs across the subsurface (see in Figure 2c and 7b). When comparing the density vs depth of the LABs to the location of the HABs (see Figure 10), it is obvious the HABs are positioned near the upper boundary of the LABs where the LABs are most dense which supports the claim that HABs originates from dense LABs.

The growth of the HABs in relation to the LABs (see in Figure 6) shows a rapid escalation in HAB growth once the LAB saturation level is achieved. Looking at the structures of individual HABs, they are not as perfectly parallel as previously proposed $[5,12,18]$, instead they vary in thickness and orientation across its length as shown in Figure 13. Figure 13c shows the start of the HABs obtained from the serial sectioning where Figures 13c2-4 shows individual white etching matter corresponding to individual LABs that later grow and join together leading to a single $\mathrm{HAB}$ shown in Figure 13c9. It can also be observed from Figure 13a5 that in some cases, HABs do not have a sharp smooth boundary, instead it appears the white etching matter of the HAB to be inclined towards LABs at the HAB boundary which affirms the relation between the two features. It is therefore reasonable to assume the observed bend in HABs (see Figure 13a) is due to the position of LABs (causing the HAB initiation) not being fully aligned vertically. As mentioned in Figure 7a, the LAB density initially is not uniform as some 
local regions are denser than others. Given the HABs stems from such dense local areas, it is reasonable to assume the initial HABs growing would vary in thickness and orientations as it grows from dense LAB local areas to relatively less dense areas. While HABs that initiate later in the bearing life will grow under a more uniform LAB structure (Figure $7 \mathrm{~b}$ ) and hence may not fluctuate in dimensions and orientations as much. The HAB shown in Figure 13a had the largest span of all HABs observed through serial sectioning (red HAB in Figure 12) which suggests it may have initiated from the early stages to support this theory. It has been hypothesised by Polonsky et al. [32] that the HAB formation may be controlled by the cyclic plasticity induced dissolution of the lenticular carbide from LABs which acts favorably at an angle of $45^{\circ}$ to the LAB. Nonetheless, further examination of early stage HABs through scanning electron microscopy is needed to confirm this theory.

Conducting the serial sectioning of the WEB network has for the first time, provided a full overview on the structure of the 3D structure of fully developed HABs compared to the LABs and inclusions within the same volume. While Figure 6 does suggest VHC steel impedes the growth of LABs and hence HABs to some extent, serial sectioning has not revealed significant interactions between the WEBs and inclusions suggesting limited influence of non-metallic inclusions. Both $\mathrm{HC}$ and VHC steels have a similar chemical composition, microstructure and hardness (average hardness of $757 \pm 9 \mathrm{HV}$ and $753 \pm 4 \mathrm{HV}$ for $\mathrm{HC}$ and VHC samples respectively) due to the similar heat treatment process applied to both steels. Given non-metallic inclusions have limited influence on WEB formation, the reason why VHC steel delays WEB formation is not clear and hence, further investigation into the micro and nanostructures and possibly residual stress distribution in needed to examine this. While majority of literature report HABs to be generally longer $[5,8,10,12,16,17]$, the serial sectioning of fully developed HABs compared to multiple LABs has suggests that the average length of both bands to be similar, although the HAB is thicker on average and have a significantly larger span (see Table 4) which can exceed a third of the contact width. Based on the 3D dimensions of the LABs and HABs obtained from serial sectioning, a simplified model of the WEB network is shown in Figure 16 to give a realistic overview on the WEB network, a schematic of the DER is also drawn based on the appearance of DER in both axial and circumferential 
cross sections.

a)
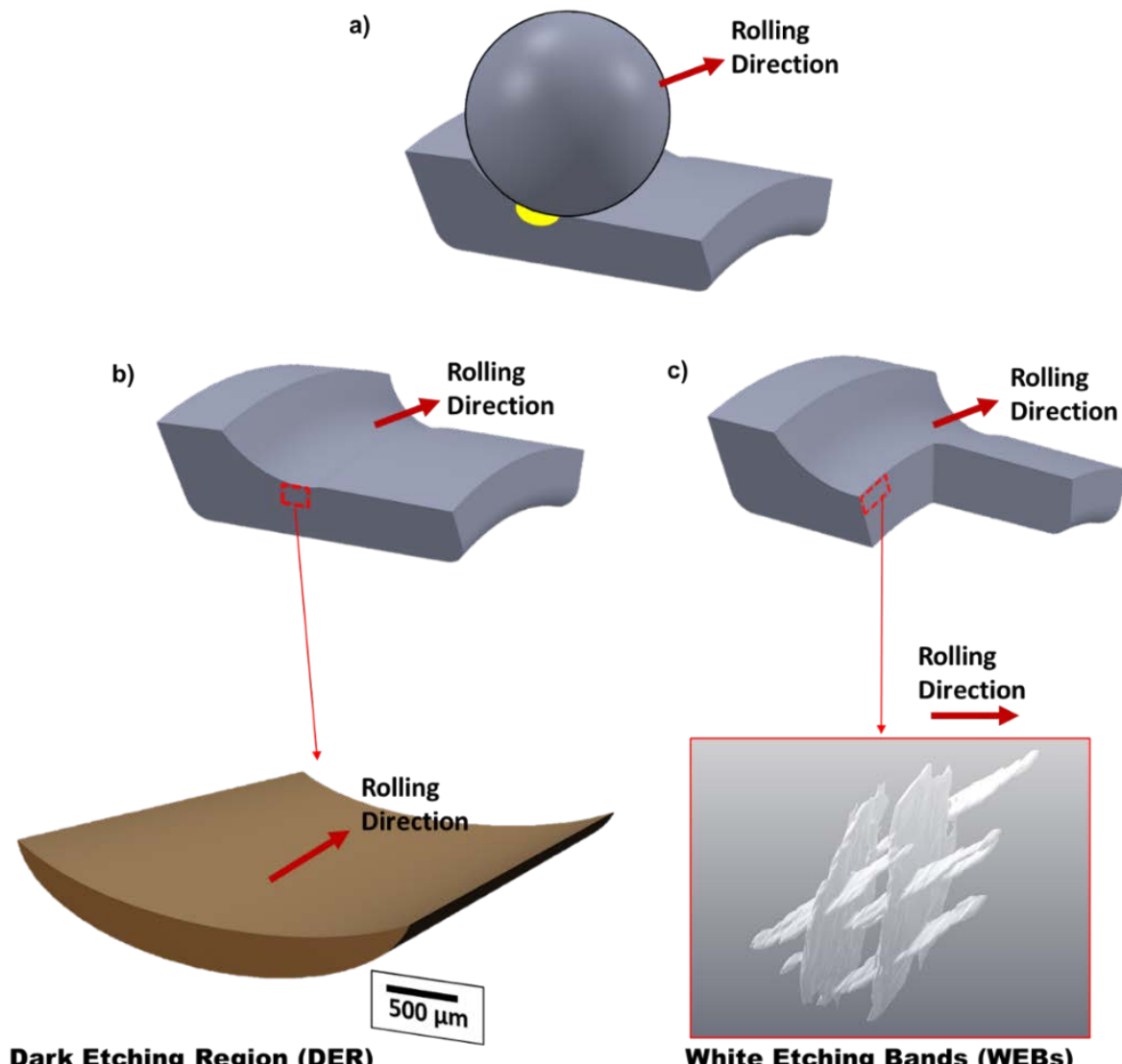

Dark Etching Region (DER)

White Etching Bands (WEBs)

Figure 16: a) position of ball and bearing inner ring and resulting stressed region (yellow) due to the contact ellipse. The 3D structure of a) DER and b) WEBs (LABs and HABs) based on dimensions in Table 5 relative to their position in the bearing inner ring within the stressed region.

\section{Conclusion}

Using various characterisation techniques, this study has provided a systematic study on the characteristics and evolution of dark etching regions (DER) and white etching bands (WEBs) which is divided into low angle bands (LABs) and high angle bands (HABs). The main conclusions from this study are:

- The thickness of the region where DER/WEBs form in the ACBB inner ring increases with contact pressure and stress cycles while the lower boundary appears to saturate later across the bearing life compared to the upper boundary. 
- The position of the WEBs correlates well with the distribution of the principal shear stress and von Mises stress whereas the DER (or only a segment of DER) is observed to be below the WEBs. The existence of WEBs within the sample overlaps the DER and hinders the detection of the upper boundary of the DER.

- The growth of LABs saturates between 1500-2000 million cycles under 2.9 GPa when the growth rate of HABs is found to increase rapidly. Evidence in this study proves the initiation and growth of HABs is dependent on the density of the pre-existing LABs. While higher contact pressure accelerates the formation of WEBs, the impact of steel cleanliness remains unclear. While the density analysis of the WEBs showed LAB development to be slightly impeded in the cleaner steel, no direct correlation between WEBs and inclusions has been found.

- $\quad$ 3-D modelling of the HABs has confirmed for the first time, that HABs are parallel discs which vary in length $(10-80 \mu \mathrm{m})$ and thickness $(1-10 \mu \mathrm{m})$ while the span of the HABs across the contact area spans from $25 \mu \mathrm{m}$ to over $575 \mu \mathrm{m}$.

- Fully developed LABs appear to have an average length similar to that of the HAB, but have a lower thickness and span in comparison. Looking at the edges of the HAB span, it appears white etching matter from LABs grow in the HAB direction which later joins together forming a single HAB.

- The serial sectioning and 3D modelling of WEBs have shown the LABs to be thin parallel plates while the HABs in the axial cross section appear as irregular white blocks in due to their thicker dimensions and orientation. The significant variation of HAB thickness and orientation is believed to attributed to the initial LAB density distribution at the early stage where HABs formed from the early stages will demonstrate more non-uniform variations compared to HABs formed in later stages.

\section{Acknowledgements}

This research has been co-funded by ESPRC (EP/N509747/1) and Schaeffler Technologies AG \& Co. KG, Schweinfurt, Germany.

\section{References}


[1] A. B. Jones, Metallographic Observations of Ball Bearing Fatigue Phenomenom, Philadelphia, 1947.

[2] H. S. Mitamura and H. Takaki, "Microstructural development in bearing steel during rolling contact fatigue," Materials Science Forum, pp. 4225-4260, 2007.

[3] H. Muro and N. Tsushima, "Microstructural, microhardness and residual stress changes due to rolling contact," Wear, vol. 15, pp. 309-330, 1970.

[4] R. Österlund and O. Vingsbo, "Phase changes in fatigued ball bearings," Metallurgical Transactions A, vol. 11, pp. 701-707, 1980.

[5] H. P. C. O. Swahn, P. C. Becker and O. Vingsbo, "Martensite decay during rolling contact fatigue in ball bearings," Metallurgical transactions A, vol. 7, pp. 1099-1110, 1976.

[6] A. P. Voskamp, "Microstructural stability and bearing performance," in Bearing Steel Technology, ASTM International, 2002.

[7] H. Fu, W. Song, E. I. Galindo-Nava and P. E. J. Rivera-Díaz-del-Castillo, "Strain-induced martensite decay in bearing steels under rolling contact fatigue: modelling and atomic-scale characterisation," Acta Materialia, vol. 139, pp. 163-173, 2017.

[8] A. Warhadpande, F. Sadeghi and R. D. Evans, "Microstructural alterations in bearing steels under rolling contact fatigue Part 1-Historical overview," Tribology Transactions, vol. 56, pp. 349-358, 2013.

[9] N. H. Forster, L. Rosado, W. P. Ogden and H. K. Trivedi, "Rolling contact fatigue life and spall propagation characteristics of AISI M50, M50 NiL, and AISI 52100, Part III: metallurgical examination," Tribology Transactions, vol. 53, pp. 52-59, 2009.

[10] J.-H. Kang, B. Hosseinkhani and P. E. J. Rivera-Díaz-del-Castillo, "Rolling contact fatigue in bearings: multiscale overview," Materials Science and Technology, vol. 28, pp. 44-49, 2012.

[11] G. Vasilca and V. Raszillier, "A study of dark etching area (D.E.A.) type structure modification of material and hertzian contact area induced by ball bearing type motion," Wear, vol. 19, no. 1, pp. 1-15, 1972.

[12] A.Voskamp, "Material Response to rolling contact loading," J Tribol, vol. 107, pp. 359-364, 1985.

[13] G. T. Hahn, V. Bhargava and Q. Chen, "The cyclic stress-strain properties, hysteresis loop shape, and kinematic hardening of two high-strength bearing steels," Metallurgical Transactions A, vol. 21, p. 653, 1990.

[14] F. Sadeghi, B. Jalalahmadi, T. S. Slack, N. Raje and N. K. Arakere, "A review of rolling contact fatigue," Journal of tribology, vol. 131, p. 041403, 2009.

[15] J. J. Bush, W. L. Grube and G. H. Robinson, "Microstructural and Residual Stress Changes in Hardened Steel due to Rolling Contact," Trans. ASM, vol. 54, pp. 390-412, 1961. 
[16] J. A. Martin, S. F. Borgese and A. D. Eberhardt, "Microstructural alterations of rolling-bearing steel undergoing cyclic stressing," Journal of Basic Engineering, vol. 88, pp. 555-565, 1966.

[17] V. Šmeḷova, A. Schwedt, L. Wang, W. Holweger and J. Mayer, "Electron microscopy investigations of microstructural alterations due to classical Rolling Contact Fatigue (RCF) in martensitic AISI 52100 bearing steel," International Journal of Fatigue, vol. 98, pp. 142-154, 2017.

[18] J. Kang, "Mechanisms of microstructural damage during rolling contact fatigue of bearing steels," 2014.

[19] V. Šmelova, A. Schwedt, L. Wang, W. Holweger and J. Mayer, "Microstructural changes in white etching cracks (WECs) and their relationship with those in dark etching region (DER) and white etching bands (WEBs) due to rolling contact fatigue (RCF)," International Journal of Fatigue, vol. 100, pp. 148-158, 2017.

[20] T. Lund, "STRUCTURAL ALTERATIONS IN FATIGUE-TESTED BALL- BEARING STEEL," Jernkontorets Ann, vol. 153, pp. 337-343, 1969.

[21] H. Fu, "Microstructural alterations in bearing steels under rolling contact fatigue," 2017.

[22] H. Fu and P. E. J. Rivera-Díaz-del-Castillo, "Evolution of White Etching Bands in 100Cr6 Bearing Steel under Rolling Contact-Fatigue," Metals, vol. 9, p. 491, 2019.

[23] H. K. D. H. Bhadeshia, "Steels for bearings," Progress in materials Science, vol. 57, pp. 268-435, 2012.

[24] A. T. W. Barrow, J.-H. Kang and P. E. J. Rivera-Díaz-del-Castillo, "The $\varepsilon \rightarrow \eta \rightarrow \theta$ transition in $100 \mathrm{Cr} 6$ and its effect on mechanical properties," Acta materialia, vol. 60, pp. 2805-2815, 2012.

[25] N. Maharjan, W. Zhou and Y. Zhou, "Micro-structural study of bearing material failure due to rolling contact fatigue in wind turbine gearbox," in Proceedings of the international symposium on current research in hydraulic turbines, Kathmandu University, Dhulikhel, Nepal, 2016.

[26] A. P. Voskamp, R. Österlund, P. C. Becker and O. Vingsbo, "Gradual changes in residual stress and microstructure during contact fatigue in ball bearings," Metals Technology, vol. 7, pp. 14-21, 1980.

[27] J. M. Beswick, "Measurement of C Levels in Structurally Transformed SAE 52100 Ball-Bearing Steel by Microprobe Analysis," Praktische Metallographie, vol. 12, pp. 200-206, 1975.

[28] S. Turteltaub and A. S. J. Suiker, "Transformation-induced plasticity in ferrous alloys," Journal of the Mechanics and Physics of Solids, vol. 53, pp. 1747-1788, 2005.

[29] S. Ganti, B. Turner, M. Kirsch, D. Anthony, B. McCoy, H. Trivedi and V. Sundar, "Three-dimensional (3D) analysis of white etching bands (WEBs) in AISI M50 bearing steel using automated serial sectioning," Materials Characterization, vol. 138, pp. 11-18, 2018.

[30] J.-H. Kang, B. Hosseinkhani, R. H. Vegter and P. E. J. Rivera-Díaz-del-Castillo, "Modelling dislocation 
assisted tempering during rolling contact fatigue in bearing steels," International Journal of Fatigue, vol. 75, pp. 115-125, 2015.

[31] N. G. Popinceanu, E. Diaconescu and S. Cretu, "Critical stresses in rolling contact fatigue," Wear, vol. 71, pp. 265-282, 1981.

[32] I. A. Polonsky and L. M. Keer, "On white etching band formation in rolling bearings," Journal of the Mechanics and Physics of Solids, vol. 43, pp. 637-669, 1995.

[33] O. Zwirlein and H. Schlicht, "Rolling contact fatigue mechanisms-accelerated testing versus field performance," in Rolling contact fatigue testing of bearing steels, ASTM International, 1982.

[34] J. Lyman, "A shear-stress normal-strain cycle relation to fatigue in bending, torsion, and rolling contact," Journal of Lubrication Technology, vol. 92, pp. 567-571, 1970.

[35] K. L. Johnson, Formation of shear bands in ball-bearing races, University of Cambridge Department of Engineering, 1988.

[36] M. El Laithy, L. Wang, T. J. Harvey, B. Vierneusel, M. Correns and T. Blass, "Further understanding of rolling contact fatigue in rolling element bearings-a review," Tribology International, p. 105849, 2019.

[37] S. Borgese, "A study of the growth mechanism of lenticular carbides in cyclically stressed 52100 steel," Journal of Lubrication Technology, vol. 92, pp. 54-58, 1970.

[38] J. Buchwald and R. W. Heckel, "An analysis of microstructural changes in 52100 steel bearings during cyclic stressing(Microstructural changes in 52100 steel bearing inner rings during cyclic stressing, obtaining thickening rate data on white-etching regions and lenticular carbides)," ASM Transactions Quarterly, vol. 61, pp. 750-756, 1968.

[39] H. Fu and P. E. J. Rivera-Díaz-del-Castillo, "A unified theory for microstructural alterations in bearing steels under rolling contact fatigue," Acta Materialia, vol. 155, pp. 43-55, 2018.

[40] H. Fu, E. I. Galindo-Nava and P. E. J. Rivera-Díaz-del-Castillo, "Modelling and characterisation of stressinduced carbide precipitation in bearing steels under rolling contact fatigue," Acta Materialia, vol. 128, pp. 176-187, 2017. 